VERSION MARCH 1, 2022: FM

Preprint typeset using $\mathrm{LAT}_{\mathrm{E}} \mathrm{X}$ style emulateapj v. 5/2/11

\title{
SPATIAL STRUCTURES IN THE GLOBULAR CLUSTER DISTRIBUTION OF THE TEN BRIGHTEST VIRGO GALAXIES
}

\author{
R. D'Abrusco ${ }^{1}$, G. Fabbiano ${ }^{1}$, A. Zezas Zez $^{1,2,3}$ \\ version March 1, 2022: $\mathrm{fm}$
}

\begin{abstract}
We report the discovery of significant localized structures in the projected two-dimensional (2D) spatial distributions of the Globular Cluster (GC) systems of the ten brightest galaxies in the Virgo Cluster. We use catalogs of GCs extracted from the HST ACS Virgo Cluster Survey (ACSVCS) imaging data, complemented, when available, by additional archival ACS data. These structures have projected sizes ranging from $\sim 5^{\prime \prime}$ to few arc-minutes $(\sim 1$ to $\sim 25 \mathrm{kpc})$. Their morphologies range from localized, circular, to coherent, complex shapes resembling arcs and streams. The largest structures are preferentially aligned with the major axis of the host galaxy. A few relatively smaller structures follow the minor axis. Differences in the shape and significance of the GC structures can be noticed by investigating the spatial distribution of GCs grouped by color and luminosity. The largest coherent GC structures are located in low-density regions within the Virgo cluster. This trend is more evident in the red GC population, believed to form in mergers involving late-type galaxies. We suggest that GC over-densities may be driven by either accretion of satellite galaxies, major dissipationless mergers or wet dissipation mergers. We discuss caveats to these scenarios, and estimate the masses of the potential progenitors galaxies. These masses range in the interval $10^{8.5}-10^{9.5} M_{\odot}$, larger than those of the Local Group dwarf galaxies.
\end{abstract}

\section{INTRODUCTION}

The large scale distribution of GCs has been studied in depth in several elliptical galaxies. Radially, GCs are known to extend to larger radii than the diffuse stellar light of the galaxies (Harris 1986), with the most luminous galaxies having relatively more centrally concentrated GCs distributions (Ashman \& Zepf 1998). The radial profiles of red (metal-rich) and blue (metal-poor) GCs differ, with red GCs matching more closely the light profile of the stellar spheroid - except for central flattened cores in the GC distribution - and blue GCs presenting a flatter extended distribution (Rhode \& Zepf 2001; Dirsch et al. 2003; Bassino et al. 2006; Mineo et al. 2014). Studies of the 2D distributions of GC systems show that the ellipticity and position angles are consistent, on average, with those of the stellar spheroid of the host galaxy (e.g. NGC4471 in Rhode \& Zepf 2001, NGC1399 in Dirsch et al. 2003 Bassino et al. 2006 NGC3379, NGC4406 and NGC4594 in Rhode \& Zepf 2004. NGC4636 in Dirsch et al. 2005, multiple galaxies in Hargis \& Rhode 2012, NGC3585 and NGC5812 in Lane et al. 2013, NGC4621 in Bonfini et al. 2012 and D'Abrusco et al. 2013). However, more recent work using the large sample of ACSVCS galaxies (Wang et al. 2013) has uncovered highly anisotropic azimuthal distributions of both red and blue GCs; these GCs are preferentially aligned with the major axis in host galaxies with significant elongation and intermediate to high luminosity.

These characteristics of the GC systems provide in-

\footnotetext{
${ }^{1}$ Harvard-Smithsonian Astrophysical Observatory, 60 Garden Street, Cambridge, MA 02138, USA

${ }^{2}$ Physics Department \& Institute of Theoretical \& Computational Physics, University of Crete, 71003 Heraklion, Crete Greece

${ }^{3}$ Foundation for Research and Technology-Hellas, 71110 Heraklion, Crete, Greece
}

sight on the evolution of the host galaxy, as well as on the formation of GCs. The relative importance of dissipative merging or of general galaxy collapse has been debated in the literature, mostly based on analytical models and observational inferences (Searle \& Zinn 1978. see Ashman \& Zepf 1992 and references therein; Forbes et al. 1997; Bekki \& Shioya 1997). Recently, N-body smoothed particle hydrodynamics simulations Bekki \& Shioya 1998, Bekki et al. 2002) have reproduced the general radial spatial distributions and metallicity properties of red and blue GC systems (but not the observed ratio of red and blue GCs) under the assumption that the elliptical galaxy was the result of major dissipative merging, as long as the merging event happened early enough in the life of the galaxy. Tidal stripping and GC accretion in interacting elliptical galaxies could help reconcile the simulations with the data (Bekki et al. 2003). Dissipationless merging of spirals could also give rise to these GC systems, but should retain kinematic signatures (Bekki et al. 2005).

While the results discussed above still do not provide a unique scenario of GC and galaxy formation, it is clear that both major merging (dissipative and more likely dissipationless) and tidal interactions play important roles. More recently, localized 2D inhomogeneities and streams have been found in GC systems of elliptical galaxies, emphasizing the role of minor merging and companion galaxy tidal disruption and accretion. These results include a spatially extended spiral-arm shaped over-density in the spatial distribution of GCs in NGC4261 (Bonfini et al. 2012, D'Abrusco et al. 2013), and more localized anisotropies in NGC4649 and NGC4278 (D'Abrusco et al. 2014a b). These features were observed within the $D_{25}$ isophotal contour of the host galaxy (1.3-4.5 $r_{e}$ ), using uniform and complete GC samples from Hubble Space Telescope (HST) observations. At larger galactocentric radii, streams in the GC distributions have 
been observed with ground-based coverage in M87 (Romanowsky et al. 2012), NGC4365 (Blom et al. 2012b) and NGC4278 (D'Abrusco et al. 2014b).

In this fourth paper in our series (D'Abrusco et al. 2013. 2014a b), we investigate how common these 2D anisotropies and streams of GC may be, by studying the 2D GC distributions of the ten brightest galaxies in the Virgo cluster observed in the ACSVCS survey (Section 2). This sample provides a unique catalog of GCs in the central regions of massive Virgo early-type galaxies. As in our previous works, we analyze the $2 \mathrm{D}$ distribution of GC systems in elliptical galaxies with a K-Nearest-Neighbor (KNN) technique supplemented by Monte Carlo simulations.

The Virgo cluster is close enough to permit the detection of GCs with HST imaging, down to relatively low flux levels with limited integration times. While the dense central environment of Virgo may discourage accretion of companions, Virgo provides a range of local densities for its galaxies. Even in its densest environment there is observational evidence of accretion of a companion galaxy, suggested by the kinematically detected GC shell in M87 (Romanowsky et al. 2012). With our sample, we can therefore begin to investigate observationally the past accretion history of galaxies in a range of environments, using the GC population as a potential marker of these events. Moreover, our method (D'Abrusco et al. 2013 is more accurate for larger samples of GCs, such as those of the more luminous elliptical galaxies of the Virgo cluster (Harris \& van den Bergh 1981; Brodie \& Strader 2006, Peng et al. 2008; Georgiev et al. 2010).

The paper is structured as follows: in Section 2 and Section 3 we describe the data and the method. In Section 4 we present the results of the analysis of the spatial distribution of GCs of each individual Virgo galaxy. In Section 5 we discuss the results obtained, focusing on the spatial distribution of the GCs structures, and we infer the properties of accreted companion galaxies that could have originated the observed GCs over-densities. We summarize our results in Section 6. We have used galaxy distances from Blakeslee et al. (2009).

\section{THE DATA}

We used data from the observations of the ten brightest galaxies in the HST Advanced Camera for Surveys (ACS) Virgo Cluster Survey (ACSVCS Côté et al. 2004), which observed the central regions of the 100 brightest early-type galaxies in the Virgo cluster, although with incomplete spatial coverage. For two galaxies (NGC4649 and NGC4365) we were able to use additional HST data to cover the full area of the galaxy.

The ACSVCS galaxies were observed in two bands, with the F475W and F850LP HST ACS filters, corresponding to the $g$ and $z$ Sloan Digital Sky Survey filters. Seven of the galaxies in our sample were covered with a single central ACS pointing; the NGC4472 observations were arranged in a triangle-shaped three-pointing mosaic reaching the $D_{25}$ elliptical isophote along the major axis. NGC4649 and NGC4365 were covered with a single pointing in the ACSVCS, but subsequent observations produced full ACS coverage (Strader et al. 2012 Blom et al. 2012b). The GC catalogs for our work were extracted from Jordán et al. (2009), which includes 12763 bona fide $\mathrm{GC}$ candidates, identified on the basis of their photometric properties and spatial extent (Jordán et al. 2004).

The number of GCs in the ACSVCS catalog associated to the ten brightest Virgo galaxies is 7053, representing $\sim 55 \%$ of the total Jordán et al. (2009) catalog. This number represents the fraction of the GC population of these galaxies that could be detected in ACSVCS observations. The basic information about the ten brightest galaxies in the ACSVCS list of targets are summarized in Table 1. Visual magnitudes $B_{T}$, numbers of GC candidates and optical morphology are all based on ACSVCS data (see Côté et al. 2004, Jordán et al. 2009; Ferrarese et al. 2006, for $B_{T}$ magnitudes, GC numbers and morphology, respectively). The details of the additional data used for NGC4649 and NGC4365 are given below.

NGC4649: a joint Chandra-HST program (P.I. Fabbiano) completed the single central ACSVCS pointing with a mosaic of five additional ACS pointings in the same F475W and F850LP filters, with comparable integration times. The final mosaic covers the whole area of the galaxy comprised within the $D_{25}\left(\sim 4.5 r_{e}\right)$, with the exclusion of the region occupied by the companion spiral galaxy NGC4647 (i.e. excluding 15\% of the NGC4649 area). From these data, Strader et al. (2012) extracted a catalog of $1516 \mathrm{GC}$ candidates that was used to characterize the spatial distribution of the GCs in NGC4649 (D'Abrusco et al. 2014a).

NGC4365: Blom et al. (2012b) used six separate ACS pointings in the same F475W and F850LP (P.I. Sivakoff) publicly available on the Hubble Legacy Archive to complement the single central ACSVCS pointing, and produced a catalog of 2163 GCs covering the entire galaxy out to $\sim 1.3 D_{25}\left(\sim 3 r_{e}\right)$. The magnitudes and sizes of the GCs in the central pointing measured by Blom et al. (2012b) are compatible with the same properties from Jordán et al. (2009). Here we use the catalog of GCs extracted by Blom et al. (2012b).

For each galaxy in Table 1, we investigate the spatial distribution of the entire sample of GCs for each galaxy and of color and luminosity classes, following the procedure outlined by D'Abrusco et al. (2013). The red and blue GC classes were determined by fitting the histogram of the $g-z$ color distribution with a double Gaussian model, and choosing the threshold corresponding to the $50 \%$ probability for a GC to belong to the red or blue class. Although for some of the galaxies the color histograms are better fit by a single Gaussian model, we adopted the color threshold based on the doubleGaussian fit to define the color classes in all cases.

For our analysis, we have also considered a split of the GC samples in luminosity using, for all galaxies, the fixed magnitude threshold $g \leq 23 \mathrm{mag}$, corresponding to an average absolute magnitude $<M_{g}^{\text {thresh }}$ ranging between $\sim-7.9$ and $\sim-8.3 \mathrm{Mag}$ (see Table 2 for the absolute magnitude threshold for each galaxy). These luminosity thresholds produce high-L and low-L GC subsamples of similar size for all the galaxies in our sample. While setting the brightness thresholds as the turnoff luminosity on the GC luminosity function may be more physically meaningful, as it would correspond to a mass threshold, that choice would produce highly unbalanced high-L and low-L classes, making our analysis statistically unreliable. The definitions used to define color and 


\begin{tabular}{|c|c|c|c|c|c|c|c|c|c|c|c|c|c|c|}
\hline \multicolumn{15}{|c|}{$\begin{array}{c}\text { TABLE } 1 \\
\text { Properties OF THE TEN BRIGHTEST VIRGO CLUSTER GALAXIES. }\end{array}$} \\
\hline \multirow[t]{2}{*}{ Galaxy } & $B_{T}$ & $D_{L}[\mathrm{Mpc}]$ & $\begin{array}{l}\% D_{25} \text { area } \\
\text { covered }\end{array}$ & $N_{\text {Tot }}$ & {$\left[N_{\text {red }}, N_{\text {blue }}\right]$} & {$\left[N_{\text {HighL }}, N_{\text {LowL }}\right]$} & $\begin{array}{l}\text { Optical } \\
\text { morphology }\end{array}$ & $\mathrm{re}_{\mathrm{e}}\left[{ }^{\prime \prime}\right]$ & $D_{25}\left[\mathrm{re}_{\mathrm{e}}\right]$ & $\left.\begin{array}{c}\Delta \text { (R.A.) } \\
{[0]}\end{array}\right]$ & $\Delta($ Dec. $)$ & $\begin{array}{l}(g-z)^{\text {thresh }} \\
{[\mathrm{mag}]}\end{array}$ & $\begin{array}{c}M_{g}^{\text {thresh }} \\
{[\mathrm{Mag}]}\end{array}$ & $\begin{array}{c}M_{\lim }(G C s) \\
{[\mathrm{Mag}]}\end{array}$ \\
\hline & $\begin{array}{l}\text { (a) } \\
9.31\end{array}$ & (b) & $\begin{array}{c}\text { (c) } \\
\sim 35 \%\end{array}$ & $\frac{(d)}{1206}$ & $\frac{(\mathrm{e})}{[708,498]}$ & $\frac{(\mathrm{f})}{7751,455}$ & $\frac{(\mathrm{g})}{\mathrm{E} 2 / \mathrm{S} 0_{-} 1_{-}(2)}$ & $\frac{(\mathrm{h})}{209.15}$ & (i) & $\begin{array}{c}(1) \\
\sim 0.004^{\circ}\left(14.7^{\prime \prime}\right)\end{array}$ & $\frac{(\mathrm{m})}{\sim 0.004^{\circ}\left(13.4^{\prime \prime}\right)}$ & (n) & $\frac{(0)}{-8.10}$ & $\frac{(\mathrm{p})}{-6.4,-7.3]}$ \\
\hline NGC4486 (M87) & 9.58 & 16.8 & $\sim 40 \%$ & 1745 & {$[1006,739]$} & {$[698,1047]$} & 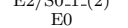 & 171.71 & $\begin{array}{l}2.9 \\
2.9\end{array}$ & $\sim 0.002^{\circ}\left(6.1^{\prime \prime}\right)$ & $\sim 0002^{\circ}\left(7.2^{\prime \prime}\right)$ & $\begin{array}{l}1.14 \\
1.21\end{array}$ & $\begin{array}{l}-8.10 \\
-8.11\end{array}$ & 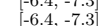 \\
\hline NGC4649 (M60) 1 & $\begin{array}{l}9.00 \\
9.81\end{array}$ & $\begin{array}{l}10.0 \\
16.6\end{array}$ & $\sim 40 \%(\sim 85 \%)$ & $807(1603)$ & {$[841,762]$} & {$[1136,467]$} & SO_1_(2) & 99.34 & 4.5 & $\sim 0.005^{\circ}\left(18.6^{\prime \prime}\right)$ & $\sim 0.004^{\circ}\left(15.7^{\prime \prime}\right)$ & 1.18 & $\begin{array}{l}-0.11 \\
-8.09\end{array}$ & $\begin{array}{l}{[-0.4,-1.0]} \\
{[-6.3,-7.2]}\end{array}$ \\
\hline NGC4406 (M86) & 10.06 & 16.4 & $\sim 40 \%$ & 367 & {$[140,227]$} & {$[218,149]$} & So 1 - $(3) / \mathrm{E} 3$ & 411.84 & $\begin{array}{l}.0 \\
1.3\end{array}$ & $\sim 0.005^{\circ}\left(18.6^{\prime \prime}\right)$ & $\sim 0.004^{\circ}\left(15.7^{\prime \prime}\right)$ & 1.15 & $\begin{array}{l}-0.09 \\
-8.26\end{array}$ & {$[-6.5,-7.4]$} \\
\hline NGC4382 (M85) & 10.09 & 17.1 & $\sim 45 \%$ & 507 & {$[283,224]$} & {$[241,266]$} & SO_1_(3) pec & 170.82 & 2.5 & $\sim 0.002^{\circ}\left(6.7^{\prime \prime}\right)$ & $\sim 0.002^{\circ}\left(5.8^{\prime \prime}\right)$ & & -8.26 & {$[-6.5,-7.4]$} \\
\hline 4 (M84) & 10.26 & 17.0 & $\sim 45$ & 506 & {$[251,255]$} & 296,2 & & 149.73 & 2.6 & $\sim 0.002^{\circ}\left(7.1^{\prime \prime}\right)$ & $\sim 0.002^{\circ}\left(6.4^{\prime \prime}\right)$ & & -8 & - 10.6, \\
\hline NGC4526 & $\begin{array}{l}10.61 \\
10.61\end{array}$ & 15.3 & $\sim 75 \%$ & 244 & {$[134,110]$} & {$[124,120]$} & S0_3_- 6 (6) & 149.10 & 2.0 & $\sim 0.002^{\circ}\left(6.9^{\prime \prime}\right)$ & $\sim 0.002^{\circ}\left(6.1^{\prime \prime}\right)$ & 1.10 & $\begin{array}{l}-0.00 \\
-8.14\end{array}$ & {$\left[\begin{array}{l}{[-0.0,-1.0]} \\
{[-6.4,-7.3]}\end{array}\right]$} \\
\hline NGC $4365^{2}$ & 10.51 & 22.0 & $\sim 45 \%(100 \%)$ & $907(2163)$ & {$[1149,1014]$} & {$[883,1280]$} & $\mathrm{E} 3$ & 116.14 & 3.6 & $\sim 0.005^{\circ}\left(17.6^{\prime \prime}\right)$ & $\sim 0.005^{\circ}\left(18.9^{\prime \prime}\right)$ & 1.16 & -8.82 & {$[-7.1,-8.0]$} \\
\hline NGC4621 (M59) & 10.76 & 15.7 & $\sim 75 \%$ & 308 & {$[158,150]$} & {$[184,124]$} & $\mathrm{E} 4$ & 106.75 & 3 & $\sim 0.002^{\circ}\left(7^{\prime \prime}\right)$ & $\sim 0.002^{\circ}\left(6.3^{\prime \prime}\right)$ & 1.23 & -7.87 & {$[-6.1,-7.0]$} \\
\hline NGC4552 (M89) & 10.78 & 15.7 & $\sim 60 \%$ & 456 & {$[204,252]$} & {$[213,243]$} & S0_1_(0) & 85.93 & 3.6 & $\sim 0.002^{\circ}\left(7.4^{\prime \prime}\right)$ & $\sim 0.002^{\circ}\left(6.4^{\prime \prime}\right)$ & 1.25 & $\begin{array}{l}-1.01 \\
-8.02\end{array}$ & {$\left[\begin{array}{l}{[-0.1,-1,0]} \\
-6.3,-7.2]\end{array}\right.$} \\
\hline \\
\hline \multirow{2}{*}{\multicolumn{15}{|c|}{$\begin{array}{l}{ }^{\mathrm{a}} B_{T} \text { magnitude from Côté et al. } 2004 \text {. } \\
{ }^{\mathrm{b}} \text { Luminosity distance of the galaxy (from NED). }\end{array}$}} \\
\hline & & & & & & & & & & & & & & \\
\hline \\
\hline \multicolumn{15}{|c|}{$\begin{array}{l}\text { d total number of GC candidates for each galaxy according to the catalog of Jordán et al. 2009) } \\
\text { enmber of GCs in the red/blue classes }\end{array}$} \\
\hline \multicolumn{15}{|c|}{$\begin{array}{l}{ }^{\mathrm{f}} \text { number of GCs in the high-L/low-L classes } \\
\mathrm{g} \text { morphological classification of the host galaxy from Ferrarese et al. } 2006\end{array}$} \\
\hline \\
\hline \multicolumn{15}{|c|}{$\begin{array}{l}\text { heffective radius } r_{e} \text { of the host galaxy from Ferrarese et al. 2006) } \\
{ }^{\mathrm{i}} D_{25} \text { expressed in units of the effective radius } r_{e}\end{array}$} \\
\hline \multirow{3}{*}{\multicolumn{15}{|c|}{$\begin{array}{l}1 \text { size of pixels in the residual maps along the R.A. axis } \\
\mathrm{m} \text { size of the pixels in the residual maps along the Dec. axis }\end{array}$}} \\
\hline & & & & & & & & & & & & & & \\
\hline \multicolumn{13}{|c|}{$\begin{array}{l}\text { n threshold } g-z \text { color used to define the red and blue GC classes } \\
\text { o threshold } M_{q} \text { absolute magnitude used to separate high-L and low-L GC classes }\end{array}$} & & \\
\hline \multicolumn{15}{|c|}{$\begin{array}{l}\text { o threshold } M_{g} \text { absolute magnitude used to separate high-L and low-L GC classes } \\
\text { P Limiting absolute magnitude of the GC catalog in the } g \text { filter, based on the completeness estimated by [Jordán et al. 2007) using simulations. The limiting magnitudes are shown for two values of the half-light } \\
\text { radii } r_{h}=0.0128^{\prime \prime} \text { and } r_{h}=0.1283^{\prime \prime} \text {, assuming an intermediate background level }\left(b_{g}=0.4023 \mathrm{mag} / \operatorname{arcsec}^{2}\right) \text {. }\end{array}$} \\
\hline \multirow{2}{*}{\multicolumn{15}{|c|}{$\begin{array}{l}\text { The fraction of area covered by observations and the number of GCs in brackets have been obtained from the imaging data and GCs catalog presented by Strader et al. } 2012 \text {, consisting in the central ACSVCS } \\
\text { pointing plus five complementary HST pointings of the outskirts of the galaxy obtained through a joint Chandra-HST proposal (P.I. Fabbiano). }\end{array}$}} \\
\hline & & & & & & & & & & & \begin{tabular}{|l|l} 
Blom et al. & 2012 \\
\end{tabular} & b), consisting & in the $\mathrm{c}$ & ral ACSVC \\
\hline
\end{tabular}

luminosity classes produce somewhat redundant samples. For instance, the overlap between red and high-L classes varies between $56 \%$ to $87 \%$ according to the host galaxy. For this reason, similarities in the residual maps of these classes are to be expected. We present the results for both color and luminosity classes for the sake of completeness.

\section{METHOD}

Following D'Abrusco et al. (2013) (see also D'Abrusco et al. 2014a b), for each sample of GCs, we generated density maps using the K-Nearest Neighbor (KNN) method of Dressler (1980). These maps are based on the local distribution of GCs, i.e. on the distance of the $K$-th closest GCs from the points where the density is estimated. The density is evaluated in each knot of a regular grid covering the region of the sky where GCs are found, and is given by:

$$
D_{K}=\frac{K}{A_{D}\left(d_{K}\right)}
$$

where $K$ is the index of the nearest neighbor used to calculate the density, and $A_{D}\left(d_{K}\right)=\pi \cdot d_{K}^{2}$ is the area of the circle with radius equal to the distance $d_{K}$ of the $K$-th nearest neighbor. The uncertainty on the KNN density scales with the square root of $K$, so that the relative fractional error is inversely proportional to the square root of $K$. For this reason, the fractional accuracy of the method increases with increasing $K$ at the expense of spatial resolution. In this paper, we reconstruct the spatial distribution of GCs using values of $K$ ranging from 2 to 10 . The size of the spatial structures depends on a combination of the value of $K$ and the density of sources. Therefore, the optimal value for $K$ depends on how extended and densely populated are these structures. In our experiments we consider a range of $K$ values that are roughly proportional to the average density of sources of the samples for which the density maps are reconstructed. Moreover, very large values of $K$ would smooth over spatially smaller structures thus losing spatial information.

For all the GC distributions, we produce residual maps by subtracting smooth azimuthally homogeneous density maps from the observed density maps. These smooth maps were generated to have the radial number density profiles of the observed GCs. Using MonteCarlo simulations, we then estimate the statistical significance of each pixel in the residual map in terms of deviation from the mean value of the simulated density distribution in the same pixels (after making sure that such distribution of simulated densities can be modeled with a Gaussian). Finally, we estimate the statistical significance of spatial structures containing multiple pixels, by taking into account the spatial features of the structure, i.e. the number of member pixels and the peculiar shape of the pixel aggregation.

The density maps for all galaxies in our sample of galaxies were evaluated on a regular spatial grid with spacing varying between $\Delta($ R.A $) \sim 0.002^{\circ}\left(\sim 6^{\prime \prime}\right)$ and $\Delta($ R.A $) \sim 0.005^{\circ}\left(\sim 18^{\prime \prime}\right)$ along the R.A. axis, and $\Delta$ (Dec) $\sim 0.002^{\circ}\left(\sim 6^{\prime \prime}\right)$ and $\Delta($ R.A $) \sim 0.005^{\circ}\left(\sim 19^{\prime \prime}\right)$ along the Dec. axis. The sizes of pixels for each target (columns (i) and (l) of Table 1) were chosen to produce a similar number of pixels in the grid covering the regions occupied by the GCs in all the galaxies investigated. This strategy permits to meaningfully compare the statistical reliability and the area measured in pixels of each spatial feature for different galaxies without taking into account the different physical scales of the host galaxies. For each galaxy, we have also compared the residual maps obtained by randomly varying the pixel sizes along both axes within a $\pm 30 \%$ interval centered on the values shown in Table 1, to rule out systematic effects on the determination of the spatial features. The density in the boundary pixels was weighted according to the fraction of the pixel within the observed region.

In Appendix A we compare the KNN method with other density reconstruction techniques, and show how it is optimal for our analysis.

\section{STRUCTURES IN THE SPATIAL DISTRIBUTION OF GCS}

For each galaxy, we have performed experiments for the entire GC sample and for the red/blue and low-L/highL classes. Each experiment involved 50,000 simulations. Table 2 shows the percentage of simulated distributions 
of GCs with "extreme" number of pixels, i.e. with values exceeding those of the $90 \%$ of pixels in the observed residual map for $K=\{7,8,9\}$, for each galaxy. The $K$ values for which these fractions are small $(\leq 1 \%)$ correspond to the spatial scale at which the observed structures become statistically significant. The parameter $K$ is a measure of the expected spatial scale of the investigated structures and of the density contrast of these structures over the average local density. Higher values of $K$ are more suitable to detect structures located within the $D_{25}$ of the galaxies, where the overall density of GCs is larger, since only high-contrast structures can be reliably detected over this high-density background. Smaller $K$ values, on the other hand, are more apt at detecting structures in regions where the total number of GCs is smaller, as in the outskirts of the host galaxies. Since the data used in this paper all cover the central regions of the galaxies, we have focused on large values of $K$. For the six galaxies NGC4472, NGC4486, NGC4649, NGC4406, NGC4526 and NGC4621, we find significant inhomogeneities for $K=[9,9,8,9,8,9]$, respectively. For NGC4382 and NGC4552 we find marginally significant inhomogeneity for $K=9$. The GC systems of NGC4374 and NGC4365 are not consistent with an overall inhomogeneous distribution for the range of $K$ values explored (see the non-negligible fraction of simulated distributions with similar overall number of "extreme" pixels in column (1) in Table 2), however, even in these cases localized statistically significant structures can still be observed.

Based on the residual maps of the entire GC system evaluated for the optimal $K$ values of the six galaxies NGC4472, NGC4486, NGC4649, NGC4406, NGC4526 and NGC4621 and for $K=9$ for the remaining four galaxies, we have considered as "over-density structures" all the groups of contiguous over-dense pixels composed of at least one pixel with significance larger than $4 \sigma$. The residual maps are shown in Figures 1 through 10 . We calculate the total significance of a GC structure by taking into account the average significance of the enclosed pixels, its size and the geometry. The total significance of a GC structure expresses the probability of observing a similarly shaped GC structure with comparable size and average significance in a simulated GC density map. More details on the estimation of the total significance can be found in D'Abrusco et al. (2013). We have classified the structures based on their total significance, size in pixels and morphology.

The three classes of over-density structures are defined as follows: small structures: groups of 4 or more adjacent pixels with total significance $\geq 4 \sigma$, or groups of one, two or three adjacent pixels with total significance $\geq 5 \sigma$; medium structures: groups of 5 to 19 adjacent pixels with with total significance $\geq 4 \sigma$; large structures, defined as groups of 20 or more adjacent pixels whose total significance is $\geq 4 \sigma$. All the remaining groups of over-dense pixels not meeting the minimal requirements were discarded. We found 24 medium and 14 "large" GC over-density structures, while the total number of structures is 229 . In the following analysis, we will focus on the medium and large over-density structures because the small structures could be produced by stochastic fluctuations in the number of GCs in just one pixel. Table 3 displays the number of all the over-density structures found in the residual maps for the $K$ values in Table 2, split according to their class, and the size (in number of pixels) for the medium and large structures. Table 3 gives also the fractions of pixels of the residual maps contained in all the structures and only in the large ones. Table 4 contains the basic properties of the 38 medium and large over-density structures, including the area of the structures in pixels, the total and excess number of GCs located within each structure. In the following, we will describe the main density features observed in each galaxy, listed in order of decreasing brightness.

\section{1. $N G C 4472$}

The $K=9$ residual map of the spatial distribution of all GCs in NGC4472 (Figure 1, upper-mid panel) is characterized by a significant $(\sim 6.5 \sigma)$ "curly" overdensity structure located $\mathrm{N}$ of the center of the galaxy (structure A1). A1 lies almost entirely outside the $D_{25}$ $\left(\sim 2.9 r_{e}\right)$ but it bends inward at its eastern end, radially following the major axis to one fourth of its length; it is more prominent in the red GCs (Figure 1, upper right panel) than in the blue GCs (Figure 1, lower left panel). The high- and low-L residual maps (Figure 1 . lower mid and right panels) suggest spatial segregation, with low-L GCs mostly located in the radial section of the structure, and high luminosity GCs located outside of the $D_{25}$, not showing a clear radial dependence. We can rule out dynamical friction as cause of the observed spatial segregation since, in that case, we would have observed separation between the high-L, more massive GCs and the low-L, less massive GCs, with the former closer to center of the galaxy and the latter at larger galactocentric distances.

The smaller distinct over-density structure A4 $(\sim 4 \sigma)$ in the N-W corner could be an extension of A1. The second and third more significant density features after A1, labeled as A3 $(\sim 4.1 \sigma)$ and A2 $(\sim 4.3 \sigma)$ in the uppermid panel of Figure 1 , are located $\mathrm{N}$ and $\mathrm{W}$ of the center of the galaxy. They are visible also in the residual maps for the two color classes and the high-L GCs. The first of these structures has a radial shape pointing N-W along the major axis.

\section{2. $N G C 4486$ (M87)}

The highly inhomogeneous 2D distribution of all GCs in NGC4486 (Table 4, Figure 2), is dominated by a large over-density structure $(\mathrm{B} 1, \sim 5.8 \sigma)$, located in the $\mathrm{N}$ $\mathrm{E}$ corner of the covered area (Figure 2, upper left and mid panels). B1 is also visible, with slightly smaller significance, in the color and luminosity GC classes (Figure 2, upper-right and lower panels). The second most significant over-density structure, in the S-E corner (B2, $\sim 4.8 \sigma)$, is also observed in red and low-L GCs. Multiple over-density structures (B5, B6, B3, B7 and B4 in Figure 2, upper mid panel) follow the major axis of NGC4486, turning to the N-W corner of the field, suggesting the presence of a large scale radial structure. Four structures $(\geq 4.2 \sigma)$ are also visible in high-L GCs (Figure 2, lower mid panel). Three of these, in the N-E corner of the field, correspond to B1, B3 and B6.

Romanowsky et al. (2012) discussed two kinematically selected GC substructures in M87, one associated to the outer-halo stellar filament $\mathrm{N}-\mathrm{W}$ of the galaxy, and the 
TABLE 2

FraCtions of Simulated RESIDUAl MAPS With NUMBER of "EXTREME" PIXELS EXCEEDing the thresholdS.

\begin{tabular}{|c|c|c|c|c|c|c|}
\hline Galaxy & & $\begin{array}{l}\text { All GCs } \\
\text { (a) }\end{array}$ & $\begin{array}{l}\text { Red GCs } \\
\text { (b) }\end{array}$ & $\begin{array}{l}\text { Blue GCs } \\
\text { (c) }\end{array}$ & $\begin{array}{l}\text { High-L GCs } \\
\text { (d) }\end{array}$ & $\begin{array}{c}\text { Low-L GCs } \\
(\mathrm{e})\end{array}$ \\
\hline \multirow[t]{3}{*}{ NGC4472 (M49) } & $K=7$ & $12.1 \%(3.1 \%)$ & $13.7 \%(4.0 \%)$ & $9.2 \%(3.8 \%)$ & $9.9 \%(4.0 \%)$ & $12.4 \%(2.1 \%)$ \\
\hline & $K=8$ & $4.2 \%(0.7 \%)$ & $8.6 \%(0.5 \%)$ & $6.6 \%(0.5 \%)$ & $4.3 \%(0.2 \%)$ & $3.9 \%(1 \%)$ \\
\hline & $K=9$ & $1.1 \%(0 \%)$ & $2.3 \%(0 \%)$ & $0.9 \%(0 \%)$ & $0.1 \%(0 \%)$ & $0.3 \%(0.1 \%)$ \\
\hline \multirow[t]{3}{*}{ NGC4486 (M87) } & $K=7$ & $10 \%(2.3 \%)$ & $12 \%(4.1 \%)$ & $8.8 \%(3.5 \%)$ & $11 \%(4.4 \%)$ & $7.5 \%(4.4 \%)$ \\
\hline & $K=8$ & $3.4 \%(0.7 \%)$ & $8.7 \%(1.9 \%)$ & $6.6 \%(1.5 \%)$ & $9.1 \%(2.1 \%)$ & $1.5 \%(0.9 \%)$ \\
\hline & $K=9$ & $0.6 \%(0 \%)$ & $1.4 \%(0.2 \%)$ & $2.9 \%(0.2 \%)$ & $4.3 \%(0.1 \%)$ & $0.2 \%(0 \%)$ \\
\hline \multirow[t]{3}{*}{ NGC4649 (M60) } & $K=7$ & $3.3 \%(0.2 \%)$ & $0.2 \%(0.3 \%)$ & $1.2 \%(0 \%)$ & $3.6 \%(0.5 \%)$ & $1.9 \%(0.7 \%)$ \\
\hline & $K=8$ & $0 \%(0 \%)$ & $0 \%(0 \%)$ & $0 \%(0 \%)$ & $0 \%(0 \%)$ & $0.1 \%(0 \%)$ \\
\hline & $K=9$ & $0 \%(0 \%)$ & $0 \%(0 \%)$ & $0 \%(0 \%)$ & $0 \%(0 \%)$ & $0 \%(0 \%)$ \\
\hline \multirow[t]{3}{*}{ NGC4406 (M86) } & $K=7$ & $4.1 \%(0.5 \%)$ & $5.2 \%(0.9 \%)$ & $9.3 \%(3.1 \%)$ & $3.1 \%(0.4 \%)$ & $1.9 \%(0.4 \%)$ \\
\hline & $K=8$ & $1.4 \%(0 \%)$ & $1.7 \%(0 \%)$ & $5.5 \%(1.2 \%)$ & $0.2 \%(0 \%)$ & $0 \%(0 \%)$ \\
\hline & $K=9$ & $0 \%(0 \%)$ & $0.1 \%(0 \%)$ & $1.4 \%(0.1 \%)$ & $0 \%(0 \%)$ & $0 \%(0 \%)$ \\
\hline \multirow[t]{3}{*}{ NGC4382 (M85) } & $K=7$ & $13.4 \%(5.4 \%)$ & $12 \%(3.3 \%)$ & $18.9 \%(9.1 \%)$ & $7.9 \%(2.1 \%)$ & $9.3 \%(3.1 \%)$ \\
\hline & $K=8$ & $8.9 \%(2 \%)$ & $8.6 \%(0.8 \%)$ & $11.1 \%(3.7 \%)$ & $4 \%(0.1 \%)$ & $4.5 \%(1.2 \%)$ \\
\hline & $K=9$ & $3.3 \%(0.2 \%)$ & $2.2 \%(0 \%)$ & $9.2 \%(0.9 \%)$ & $0.2 \%(0 \%)$ & $0.6 \%(0.1 \%)$ \\
\hline \multirow[t]{3}{*}{ NGC4374 (M84) } & $K=7$ & $24.4 \%(11.2 \%)$ & $11.6 \%(7.8 \%)$ & $14.5 \%(5.5 \%)$ & $19.9 \%(8.8 \%)$ & $13.5 \%(8.1 \%)$ \\
\hline & $K=8$ & $21 \%(9.7 \%)$ & $9 \%(5.1 \%)$ & $3.9 \%(1.1 \%)$ & $12.3 \%(4.6 \%)$ & $9.7 \%(4.4 \%)$ \\
\hline & $K=9$ & $12.5 \%(2.9 \%)$ & $4.9 \%(0.8 \%)$ & $2.1 \%(0.3 \%)$ & $7.8 \%(1.9 \%)$ & $5.4 \%(3.2 \%)$ \\
\hline \multirow[t]{3}{*}{ NGC4526 } & $K=7$ & $0.1 \%(0 \%)$ & $0 \%(0 \%)$ & $0 \%(0 \%)$ & $0 \%(0 \%)$ & $0.3 \%(0 \%)$ \\
\hline & $K=8$ & $0 \%(0 \%)$ & $0 \%(0 \%)$ & $0 \%(0 \%)$ & $0 \%(0 \%)$ & $0 \%(0 \%)$ \\
\hline & $K=9$ & $0 \%(0 \%)$ & $0 \%(0 \%)$ & $0 \%(0 \%)$ & $0 \%(0 \%)$ & $0 \%(0 \%)$ \\
\hline \multirow[t]{3}{*}{ NGC4365 } & $K=7$ & $36.6 \%(25.7 \%)$ & $30.8 \%(19.5 \%)$ & $21.3 \%(14.4 \%)$ & $18.3 \%(10.4 \%)$ & $40.6 \%(31.1 \%)$ \\
\hline & $K=8$ & $31.4 \%(23.1 \%)$ & $28.2 \%(14.5 \%)$ & $15.6 \%(9.1 \%)$ & $12.7 \%(7.6 \%)$ & $26.9 \%(19.1 \%)$ \\
\hline & $K=9$ & $24.3 \%(18.9 \%)$ & $17.6 \%(8.3 \%)$ & $5.9 \%(0.9 \%)$ & $5.7 \%(0.5 \%)$ & $22.2 \%(12 \%)$ \\
\hline \multirow{3}{*}{ NGC4621 (M59) } & $K=7$ & $8.4 \%(0.4 \%)$ & $3.9 \%(0.1 \%)$ & $4.8 \%(0.5 \%)$ & $6.5 \%(1.7 \%)$ & $1.9 \%(0 \%)$ \\
\hline & $K=8$ & $1.1 \%(0 \%)$ & $0.5 \%(0 \%)$ & $2 \%(0 \%$ & $4.3 \%(0.2 \%)$ & $0.3 \%(0 \%)$ \\
\hline & $K=9$ & $0 \%(0 \%)$ & $0 \%(0 \%)$ & $0.1 \%(0 \%)$ & $0.2 \%(0 \%)$ & $0 \%(0 \%)$ \\
\hline \multirow[t]{3}{*}{ NGC4552 (M89) } & $K=7$ & $12.9 \%(7.6 \%)$ & $13.6 \%(6.5 \%)$ & $7.7 \%(1.9 \%)$ & $6.4 \%(2 \%)$ & $13.7 \%(8.8 \%)$ \\
\hline & $K=8$ & $6.5 \%(3.2 \%)$ & $9.5 \%(4.8 \%)$ & $3.6 \%(0.5 \%)$ & $3.2 \%(0.4 \%)$ & $9.5 \%(3.5 . \%)$ \\
\hline & $K=9$ & $1.9 \%(0.2 \%)$ & $3.6 \%(1.3 \%)$ & $1.2 \%(0.1 \%)$ & $0.9 \%(0 \%)$ & $2.7 \%(0.8 \%)$ \\
\hline
\end{tabular}

Column description:

a fraction of simulations obtained with $K=\{7,8,9\}$ with number of extreme pixels with density exceeding the 90-th percentile of the observed pixel residual distribution) for the entire sample of GCs.

$\mathrm{b}$ as in column (a) for red GCs only.

${ }^{\mathrm{c}}$ as in column (a) for blue GCs only.

$\mathrm{d}$ as in column (a) for high-L GCs only.

$\mathrm{e}$ as in column (a) for low-L GCs only.

other in the inner halo (the "shell"). This second structure was interpreted as the signature of a disrupted infalling system. Both phase-space structures are located outside of the region observed by ACSVCS. We have applied our method to the sample of Romanowsky et al. (2012) (see also Strader et al. 2011) to assess our ability to detect these kinematically selected structures as over-densities in the GC spatial distribution (Figure 2 lower panels). We should keep in mind, however, that the ACSVCS and the Romanowsky et al. (2012) samples have different levels of completeness; in particular, the Romanowsky et al. (2012) sample is biased towards higher luminosity (mass) GCs.

At very large radii, we find a high-significance overdensity structure W of NGC4486, located where seven GC members of the shell (green points in lower, left panel in Figure 2 can be found. While the other members of the shell are distributed at the border of under-density areas (blue), they do not constitute a statistically signif- icant overdensity. A zoomed-in version of the residual map obtained from the Romanowsky et al. (2012) GCs sample for $K=9$ (lower mid panel, Figure 2 shows that a very significant $(\sigma \sim 10)$, extended GC structure is located N and N-W of NGC4486, mostly outside the $D_{25}$ of the galaxy. A low-significance bridge of overdensity pixels connects this large GC structure to the complex of GCs over-densities observed in the ACSVCS residual map (B1, B3, B4 and B7). The average line-of-sight velocity of all Romanowsky et al. (2012) GCs enclosed within this large structure is consistent with the bulk velocity of the system within $2 \sigma$. The average velocities of the Romanowsky et al. (2012) GCs located within the ACSVCS GC structures are consistent with the bulk velocity of the system, but the numbers of GCs are small (4 Romanowsky et al. 2012 GCs in B1, 3 in B2, 2 in $\mathrm{B} 3,8$ in $\mathrm{B} 4,4$ in $\mathrm{B} 5,3$ in $\mathrm{B} 6$ and 0 in $\mathrm{B} 7$ ). For this reason, we cannot rule out a distinct kinematics, based on the Strader et al. (2011) sample only. A complete 
TABLE 3

Global PROPERTIES OF THE OVER-DENSITY STRUCTURES.

\begin{tabular}{|c|c|c|c|c|c|c|c|}
\hline Galaxy & $\begin{array}{l}\mathrm{N}_{\text {tot }} \\
\text { (a) }\end{array}$ & $\begin{array}{c}\mathrm{N}_{\text {medium }} \\
\text { (b) }\end{array}$ & $\begin{array}{c}\mathrm{N}_{\text {large }} \\
\text { (c) }\end{array}$ & $\begin{array}{l}\% \text { observed area in } \\
\text { medium }+ \text { large } \\
\text { structures }(\mathrm{d})\end{array}$ & $\begin{array}{c}\text { \% observed area in } \\
\text { large structures } \\
(\mathrm{e})\end{array}$ & $\begin{array}{l}\text { \% GCs in } \\
\text { all structures } \\
\text { (f) }\end{array}$ & $\begin{array}{l}\text { \% excess GCs in } \\
\text { all structures } \\
(\mathrm{g})\end{array}$ \\
\hline NGC4472 (M49) & 27 & 3 & 1 & $14.5 \%$ & $3.4 \%$ & $11.7 \%$ & $4.9 \%$ \\
\hline NGC4486 (M87) & 46 & 6 & 1 & $16.9 \%$ & $2.1 \%$ & $17.2 \%$ & $6.5 \%$ \\
\hline NGC4649 (M60) & 28 & 0 & 1 & $16.6 \%$ & $8.5 \%$ & $10.9 \%$ & $5.7 \%$ \\
\hline NGC4406 (M86) & 12 & 3 & 1 & $16.5 \%$ & $11.7 \%$ & $26.4 \%$ & $13.2 \%$ \\
\hline NGC4382 (M85) & 21 & 2 & 2 & $14.6 \%$ & $7.2 \%$ & $22.3 \%$ & $9.9 \%$ \\
\hline NGC4374 (M84) & 22 & 3 & 1 & $13.6 \%$ & $3.2 \%$ & $14.4 \%$ & $6.8 \%$ \\
\hline NGC4526 & 4 & 0 & 2 & $30.1 \%$ & $28.1 \%$ & $49.2 \%$ & $28.6 \%$ \\
\hline NGC4365 & 38 & 4 & 1 & $15.2 \%$ & $2.8 \%$ & $9.7 \%$ & $4.3 \%$ \\
\hline NGC4621 (M59) & 10 & 0 & 2 & $18.4 \%$ & $16.2 \%$ & $35.4 \%$ & $16.4 \%$ \\
\hline NGC4552 (M89) & 21 & 3 & 2 & $16.6 \%$ & $5.8 \%$ & $21.3 \%$ & $9.8 \%$ \\
\hline
\end{tabular}

Column description:

${ }^{\text {a }}$ number of all GC over-density structures.

${ }^{\mathrm{b}}$ number of medium GC over-density structures.

${ }^{\mathrm{c}}$ number of large GC over-density structures

$\mathrm{d}$ fraction of observed area of the galaxy belonging to medium and large GC structures.

${ }^{e}$ fraction of observed area of the galaxy belonging to the large GC over-density structures.

f percentage of GCs located within GC structures over the total number of GCs

$\mathrm{g}$ percentage of excess GCs located within GC structures over the total number of GCs
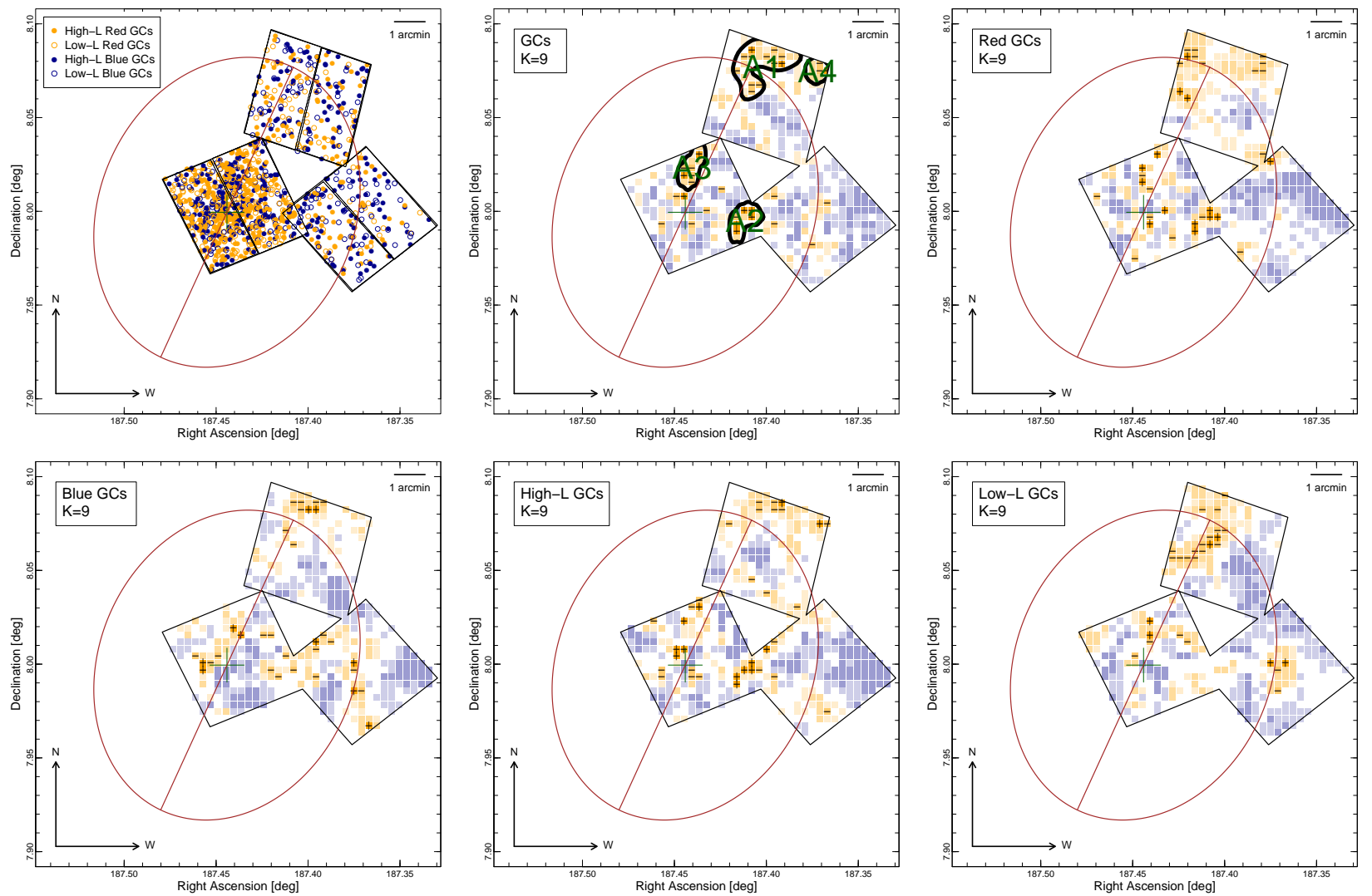

FIG. 1.- (Upper left) Scatterplot of the positions of the GCs detected in NGC4472 and used in this paper. Orange and blue points correspond to red and blue GCs, while solid and open symbols show high-luminosity and low-luminosity sources, respectively, defined using the magnitude threshold discussed in Section 2 (Upper middle) Residual map obtained for $K=9$ of the whole sample of GCs in NGC4472. Blue and orange pixels show negative and positive excess, i.e. under-density and over-density residual. The intensity and symbols express the degree of significance of each pixel: light blue/orange, dark blue/orange, horizontal bars and crosses indicate over/under-density pixels with significance smaller or equal than 1, smaller or equal than 2, smaller or equal than 3 and larger than 3, respectively. (Upper right) $K=9$ residual map for red GCs. (Lower left) $K=9$ residual map for blue GCs. (Lower middle) $K=9$ residual map for high-luminosity GCs. (Lower right) $K=9$ residual map for low-luminosity GCs. In all plots, the ellipse shows the $D_{25}\left(\sim 2.9 r_{e}\right)$ isophote of the galaxy from de Vaucouleurs et al. (1991). The solid black line shows the footprint of the HST observations used to extract the GCs. 
TABLE 4

Main Properties of the "Medium" and "LARGE" GCs OVer-Density StruCtures.

\begin{tabular}{|c|c|c|c|c|c|c|c|}
\hline Galaxy & $\begin{array}{l}\text { Structure } \\
\text { (a) }\end{array}$ & $\begin{array}{l}\text { Class } \\
\text { (b) }\end{array}$ & $\begin{array}{c}\text { Significance } \\
\text { (c) }\end{array}$ & $\begin{array}{c}\text { Size [pixels] } \\
(\mathrm{d})\end{array}$ & $\begin{array}{c}\text { Total GCs } \\
\text { (e) }\end{array}$ & $\begin{array}{l}\text { Excess GCs } \\
\text { (f) }\end{array}$ & $\begin{array}{c}\text { Physical size [kpc] } \\
(\mathrm{g})\end{array}$ \\
\hline \multirow[t]{4}{*}{ NGC4472 (M49) } & A1 & All, red, blue, high-L*, low-L* & $\sim 6.5 \sigma$ & 22 & 25 & 8.1 & 14.4 \\
\hline & A2 & All, red, blue, high-L, & $\sim 4.3 \sigma$ & 9 & 46 & 23.2 & 5.3 \\
\hline & A3 & All, red, blue, high-L, low-L & $\sim 4.1 \sigma$ & 10 & 47 & 16.6 & 4.6 \\
\hline & A4 & All, red, high-L & $\sim 4 \sigma$ & 7 & 6 & 2.1 & 1.1 \\
\hline \multirow[t]{7}{*}{ NGC4486 (M87) } & B1 & All, red, blue, high-L, low-L & $\sim 5.8 \sigma$ & 18 & 23 & 11.3 & 5.7 \\
\hline & B2 & All, red, blue, low-L & $\sim 4.8 \sigma$ & 20 & 31 & 12.1 & 1.2 \\
\hline & B3 & All, red, blue, high-L & $\sim 4.7 \sigma$ & 9 & 15 & 6.8 & 1.4 \\
\hline & B4 & All, red, blue, low-L & $\sim 4.5 \sigma$ & 9 & 83 & 38.7 & 5.8 \\
\hline & B5 & All, red, low-L & $\sim 4.5 \sigma$ & 6 & 28 & 8.8 & 1.6 \\
\hline & B6 & All, red, high-L, low-L & $\sim 4.3 \sigma$ & 7 & 25 & 12.8 & 4.6 \\
\hline & B7 & All, red & $\sim 4.1 \sigma$ & 18 & 18 & 5.6 & 1 \\
\hline NGC4649 (M60) & $\mathrm{C} 1$ & All, red, high-L*, low-L* & $\sim 10 \sigma$ & 60 & 160 & 80.3 & 29 \\
\hline \multirow[t]{4}{*}{ NGC4406 (M86) } & D1 & All, red, blue, high-L, low-L & $\sim 15 \sigma$ & 91 & 76 & 38.8 & 14.3 \\
\hline & D2 & All, blue, high-L & $\sim 4.5 \sigma$ & 7 & 4 & 1.2 & 2.4 \\
\hline & D3 & All, red, low-L & $\sim 4.3 \sigma$ & 8 & 5 & 2.4 & 4.6 \\
\hline & D4 & All, red, high-L & $\sim 4 \sigma$ & 8 & 4 & 2 & 5.1 \\
\hline \multirow[t]{4}{*}{ NGC4382 (M85) } & E1 & All, red, blue, high-L, low-L & $\sim 8.5 \sigma$ & 61 & 36 & 18.6 & 7.9 \\
\hline & $\mathrm{E} 2$ & All, red, blue, high-L* ${ }^{*}$, low-L* & $\sim 6.8 \sigma$ & 22 & 26 & 11.8 & 6.4 \\
\hline & E3 & All, red, high-L & $\sim 6.3 \sigma$ & 12 & 20 & 3.4 & 3.1 \\
\hline & E4 & All, red, blue, high-L, low-L & $\sim 5.7 \sigma$ & 19 & 19 & 9.1 & 6.2 \\
\hline \multirow[t]{4}{*}{ NGC4374 (M84) } & $\mathrm{F} 1$ & All, blue, high-L & $\sim 6.5 \sigma$ & 30 & 18 & 9.2 & 6.4 \\
\hline & $\mathrm{F} 2$ & All, red, blue, high-L, low-L & $\sim 6.3 \sigma$ & 19 & 9 & 3.2 & 3.5 \\
\hline & F3 & All, blue, high-L & $\sim 5.5 \sigma$ & 17 & 23 & 6.6 & 3.6 \\
\hline & F4 & All, red, high-L, low-L & $\sim 4.5 \sigma$ & 11 & 7 & 1.4 & 2.7 \\
\hline \multirow[t]{2}{*}{ NGC4526 } & G1 & All, red, blue, high-L, low-L & $>20 \sigma$ & 185 & 63 & 37.9 & 9.3 \\
\hline & G2 & All, red, blue, high-L, low-L & $>20 \sigma$ & 88 & 53 & 28.8 & 16.7 \\
\hline \multirow[t]{5}{*}{ NGC4365 } & $\mathrm{H} 1$ & All, red*, blue* & $\sim 5.8 \sigma$ & 22 & 72 & 29.5 & 23.2 \\
\hline & $\mathrm{H} 2$ & All, red, blue, high-L, low-L & $\sim 5.1 \sigma$ & 10 & 45 & 5.6 & 18.7 \\
\hline & H3 & All, blue, high-L, low-L & $\sim 4.6 \sigma$ & 5 & 24 & 6.2 & 11.2 \\
\hline & $\mathrm{H} 4$ & All, blue, high-L, low-L & $\sim 4.5 \sigma$ & 6 & 28 & 18.4 & 4.3 \\
\hline & H5 & All, red, blue, low-L & $\sim 4.1 \sigma$ & 6 & 24 & 12.5 & 4.2 \\
\hline \multirow{2}{*}{ NGC4621 (M59) } & $\mathrm{I} 1$ & All, red, blue, high-L, low-L & $>15 \sigma$ & 71 & 42 & 23.4 & 10.9 \\
\hline & $\mathrm{I} 2$ & All, red, blue, high-L, low-L & $>15 \sigma$ & 85 & 56 & 21.3 & 7.7 \\
\hline \multirow[t]{5}{*}{ NGC4552 (M89) } & L1 & All, blue, high-L & $\sim 8.7 \sigma$ & 30 & 7 & 4.1 & 5 \\
\hline & $\mathrm{L} 2$ & All, red, blue, low-L & $\sim 7.3 \sigma$ & 22 & 11 & 6.4 & 4.2 \\
\hline & L3 & All, red, blue, high-L, low-L & $\sim 6.4 \sigma$ & 18 & 26 & 10.8 & 4.3 \\
\hline & L4 & All, red, blue, high-L, low-L & $\sim 5.7 \sigma$ & 10 & 23 & 11.2 & 3.3 \\
\hline & L5 & All, red, high-L* ${ }^{*}$ low-L* & $\sim 4.8 \sigma$ & 17 & 16 & 8.5 & 4.2 \\
\hline
\end{tabular}

Column description:

a unique code associated to the GCs structure

b significance of each structure in the residual maps generated for all GCs, the color and luminosity GC classes: boldface text is used for the GC classes where the structure is clearly visible and has large statistical significance, plain text is used for GC classes where the structure is still detected with lower significance, while the GC classes where the structure is not detected - or detected in a distinctly different shape and size - are not reported. Asterisks indicate cases where the residual maps of either the color or luminosity GC classes suggest the presence of spatial segregation

${ }^{\mathrm{c}}$ statistical significance of the structure

d size of the structures measured in pixels

e total number of GCs within the structures

$\mathrm{f}$ number of GCs within the structures in excess to the smooth distribution of GCs used to evaluate the residual maps

$\mathrm{g}$ approximate maximum physical size of the GC structures [kpc] 
photometric coverage of M87, at least including the area within its $D_{25}$ and a deeper, more homogeneous spectroscopic campaign that can match the ACSVCS sample are needed to fully map its GC streamers and confirm their nature in the phase-space.

NGC4486 shows dust filaments (Ferrarese et al. 2006) and has been detected by di Serego Alighieri et al. (2013) in the far infrared (FIR) images at $250 \mu \mathrm{m}$ obtained by the Herschel Virgo Cluster Survey. The FIR emission indicates the presence of diffuse dust that may affect the detection of GCs and, in turn, our reconstruction of their spatial distribution. The location of the observed dust structures is not associated with significant underor over-density regions in our residual GC maps, suggesting an overall negligible effect of the dust on the the reconstruction of the GC spatial distribution obtained with our method.

\section{3. $N G C 4649$}

The residual map $(K=8)$ of the spatial distribution of all GCs detected in NGC4649 (D'Abrusco et al. 2014a) (Figure 3, upper mid panel) is dominated by a highly significant $(\sim 10 \sigma)$, spatially extended $(\sim 50$ pixels $)$ overdensity structure (C1) in the E side of the galaxy. This feature, less prominent in red GCs, originates close to the major axis, following it out to the $D_{25}\left(\sim 4.5 r_{e}\right)$ before bending northward; high-L and low-L GCs appear spatially segregated in C1 (Figure 3, lower mid and right panels), with the former mostly located along the major axis and the latter following the $D_{25}$ ellipse. The blue GCs residual map (Figure 1, lower left panel) also shows few more marginally significant over-density structures in the $\mathrm{S}$ and $\mathrm{W}$ sides of the galaxies. As discussed in D'Abrusco et al. (2014a), the GC over-density structure is not associated to any feature in the diffuse stellar light. Recent kinematic measurements Arnold et al. 2014) of the diffuse stellar light have revealed disk-like outer rotation, which suggests a major dry merger undergone by a massive lenticular galaxy progenitor. No obvious correlation between the GCs structure and the radial velocities of a sample of GCs observed spectroscopically by Lee et al. (2008) was found (D'Abrusco et al. 2014a).

\section{4. $N G C 4406$}

The $K=9$ residual map of all GCs in NGC4406 (Figure 4. upper mid panel) is dominated by a large, spatially coherent structure $(\mathrm{D} 1, \sim 15 \sigma)$ located N-E of the center of the galaxy. At small galactocentric distances, D1 follows the radial direction, but it widens as it reaches the boundary of the field. D1 is clearly visible in both color and luminosity GC classes (Figure 4. upper right and lower panels). While D1 in red GCs is mostly located along the radial direction, in blue GCs it is more azimuthally extended. The second most significant residual structure (D2, $\sim 4.5 \sigma$ ) overlaps the S-E end of the major axis. D3 and D4 $(\sim 4.3 \sigma$ and $\sim 4 \sigma$ respectively) are visible in the whole sample of GCs, to the W of the major axis. D3 is more evident in the red (Figure 4, upper right panel) and low-L (Figure 4, lower left panel) GCs, indicating the presence of a cluster of metal-rich, low-luminosity GCs at galactocentric radius $\sim 1.4^{\prime}$.

A sub-arcsecond dusty disk has been observed in NGC4406 (Ferrarese et al. 2006; di Serego Alighieri et al. 2013). Since the spatial scale of the structure that we detect in the GC distribution is significantly larger that few arcseconds, the presence of the dust feature should not affect our results.

\section{5. $N G C 4382$}

The $K=9$ spatial distribution of all GCs in NGC4382, (Figure 5, upper mid panel), is characterized by the presence of two large, significant over-density structures. E1 $(>10 \sigma)$, located in the $\mathrm{S}-\mathrm{W}$ corner of the HST field, is radial in the entire GC sample and is visible in all GC color classes (Figure 5, upper right and lower left panels). E2 $(\sim 6.8 \sigma)$, S of the center of the galaxy, crosses the major axis at $\sim 0.8^{\prime}$ from the center. E2 and E1 could be distinct sections of a single elongated structure. A second group of significant and spatially coherent structures is located $\mathrm{N}-\mathrm{W}$; these are clearly visibile in the residual maps of all and red GCs: E3 $(\sim 6.3 \sigma)$ and E4 $(\sim 5.7 \sigma)$. $\mathrm{E} 4$ looks similar to $\mathrm{E} 1$, and extends radially from the center to the $\mathrm{N}-\mathrm{W}$ corner of the field (Figure 5, upper mid panel). Another property of the spatial distribution of all GCs in NGC4382 is the very significant $(\sim 6.5 \sigma)$ E-W anisotropy, with $\sim 68 \%$ of all GCs located $\mathrm{E}$ of the major axis of the galaxy. Since the ACSVCS image is not centered on NGC4382 center, it is not surprising tat the asymmetry between the $\mathrm{E}$ and $\mathrm{W}$ equal area halves of the ACSVCS field is slightly more significant, with $\sim 72 \%$ of the total GCs located in the western half of the field.

\section{6. $N G C 4374$}

This galaxy has only one large over-density structure, F1 $(\sim 6.5 \sigma)$, located in the S-W corner of the ACSVCS data field (Figure 6, upper mid panel). F1 is not visible in the red and low-luminosity GC classes (Figure 6, upper right and lower mid panels). The smaller over-density structures F2 $(\sim 6.3 \sigma), \mathrm{F} 3(\sim 5.5 \sigma)$ and F4 $(\sim 4.5 \sigma)$ are roughly located along the major axis. F3 lies nearby F1, suggesting that they may be physically connected.

NGC4374 is known for the presence of abundant dust (di Serego Alighieri et al. 2013). In particular, two parallel dust lanes, located close to the center of the galaxy and slightly $\mathrm{N}$ of it along the $\mathrm{E}-\mathrm{W}$ direction $($ Ferrarese et al. 2006), extend for $\sim 5^{\prime \prime}$ and $\sim 14^{\prime \prime}$, respectively. While the presence of the dust lane may have affected the detection of GCs close to the position of F4, the GC structures F1, F2 and F3 are located too far from the dust to be significantly affected.

\section{7. $N G C 4526$}

We detected (Figure 7, upper mid panel) two strong spatially extended over-density structures located W (G1) and $E(G 2)$ of the center of the galaxy, both $\geq 10 \sigma$ significant and clearly visible in all the classes (Figure 7 . upper right and lower panels). G1, at small galactocentric distances $\left(<1^{\prime}\right)$, roughly follows the $W$ section of the major axis of the galaxy, and it extends towards $\mathrm{N}$ as it approaches the boundary of the field covered by the HST data, at galactocentric distance between $\sim 1^{\prime}$ and $\sim 1.6^{\prime}$. G2 overlaps the E section of the major axis.

Since NGC4526 is a lenticular (S0_3_(6)) galaxy (Ferrarese et al. 2006), the two structures G1 and G2 could be enhanced by projection effects, assuming a smooth 

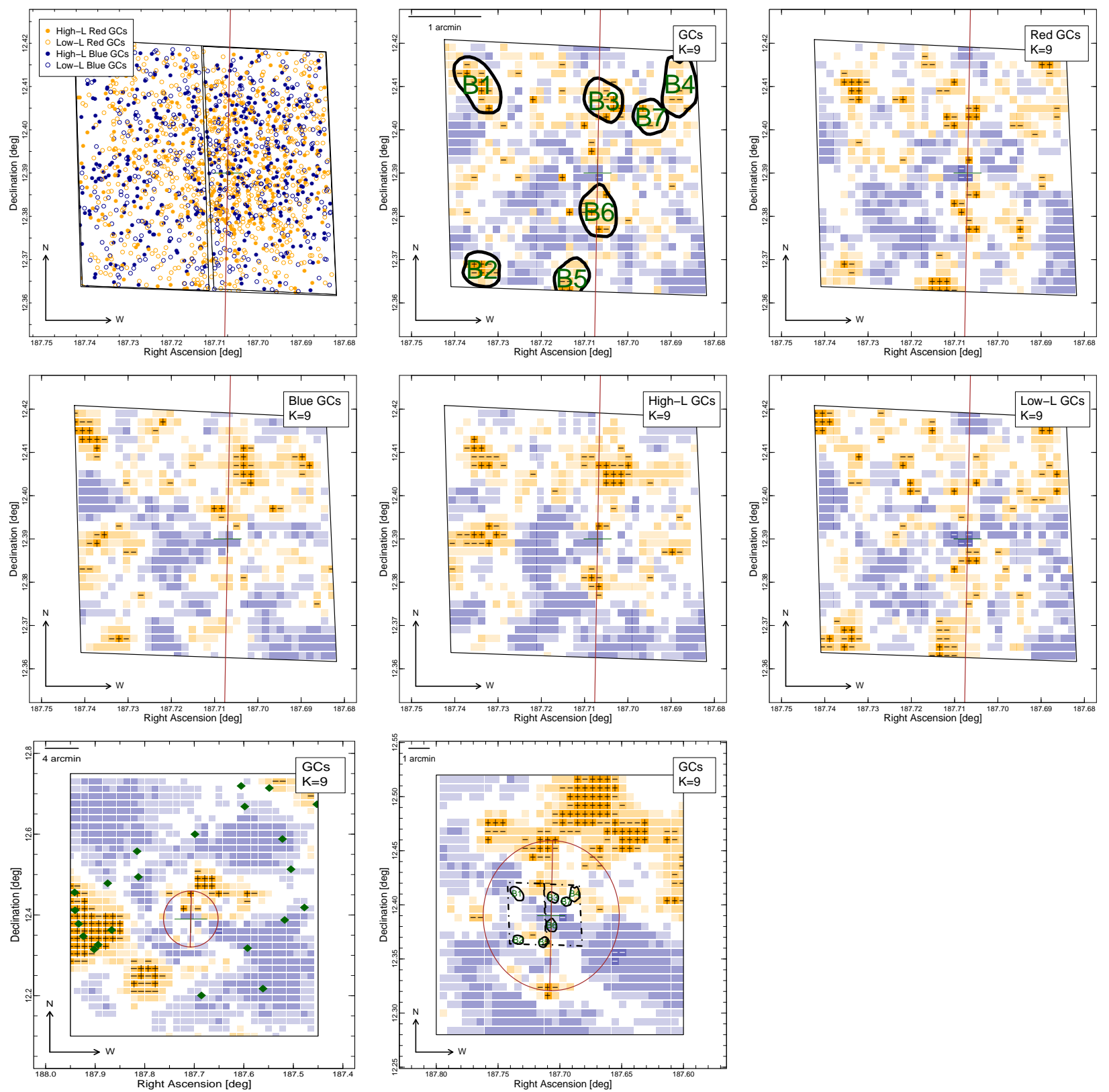

FIG. 2.- Upper and mid panels: scatterplot of the position of the GCs in NGC4486 and residual maps obtained for $K=9$ for the whole sample of GCs and the two color and luminosity GC classes. The $D_{25}$ elliptical isophote $\left(\sim 2.9 r_{e}\right)$ is not shown because it falls outside of the plot borders. The major axis of the galaxy is shown by the vertical red line. Refer to Figure 1 for a description of the other panels. Lower panels: residual map for $K=9$ for the sample of GCs observed spectroscopically (Strader et aI. 2011 Romanowsky et al.2012). The GCs belonging to the shell selected kinematically are shown as green diamonds. On the right, a zoomed-in version of the same map with the region covered by the ACSVCS data and the GC structures discussed in Section 4.2 highlighted.

three-dimensional distribution of GCs that follows the galaxy light. In order to check whether projection effect may bias our results, we have produced additional residual maps using a modified model for the simulated spatial distribution of GCs. We add to the smooth background component with isotropic azimuthal distribution (the halo) an azimuthally anisotropic component aligned along the major axis, spanning $15 \mathrm{deg}$ above and below the major axis measured at the $D_{25}$, to mimic the presence of a population of GCs associated to the disk. We have performed different experiments where the fractions of simulated GCs associated to either component varies between $30 \%$ and $70 \%$. Both G1 and G2 are still visi- ble, although with decreasing significance as the fraction of simulated GCs in the disk component grows. In the worst case scenario, the significance of G1 is $\sim 7 \sigma$ and the significance of G2 is $\sim 3.5 \sigma$. Based on the results, we can rule out that both G1 and G2 structures are entirely spurious.

Moreover, NGC4526 contains abundant dust (Ferrarese et al. 2006, di Serego Alighieri et al. 2013), mostly located in a large-scale $\left(15.6^{\prime \prime}\right)$ disk structure aligned along the major axis of the galaxy. The non-negligible dust obscuration may have affected the detection efficiency of GCs and, in turn, the characterization of their spatial structure. Spectroscopic measurements could 

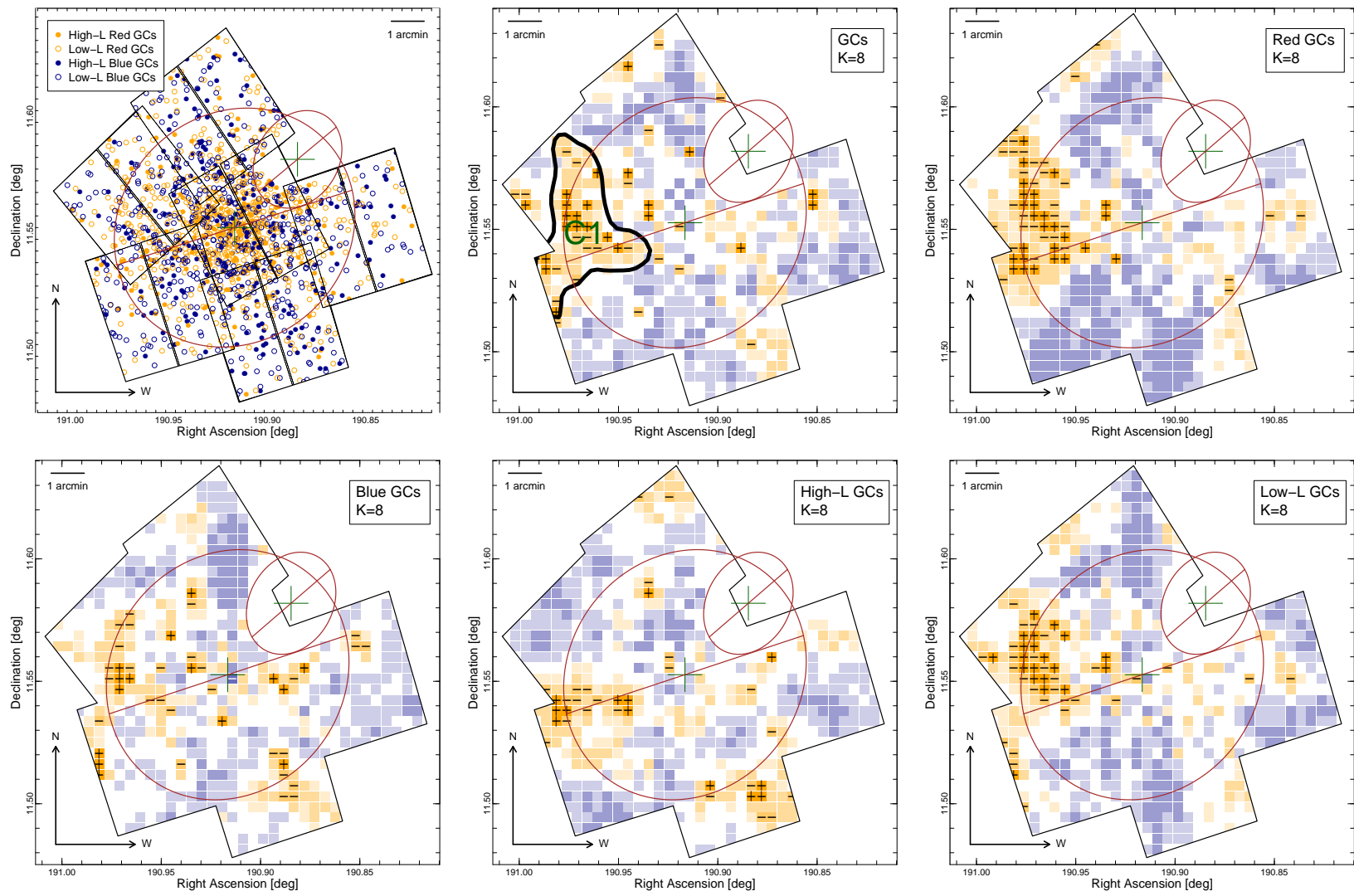

FIG. 3. - Scatterplot of the position of the GCs in NGC4649 and residual maps obtained for $K=8$ for the whole sample of GCs and the two color and luminosity classes. These plots are slightly modified versions of the plots shown by D'Abrusco et al. (2014a). Refer to Figure 1 for a description of each panel. The smaller ellipse represents the area occupied by NGC4647, a less massive spiral galaxy that is thought to be interacting with NGC4649. This area was not observed with ACS (Strader et al. 2012).

confirm the nature of G1 and G2 as physically distinct groups of GCs by detecting them also in the phase-space.

\section{8. $N G C 4365$}

The spatial distribution of all GCs detected in NGC4365 (Figure 8, upper mid panel) is characterized by the absence of large structures and by a marginally significant $(2.8 \sigma)$ asymmetry in the distribution of GCs, which are more numerous to the $\mathrm{E}$ of the major axis of the galaxy. The most significant feature H1 $(\sim 5.8 \sigma)$, is close to the intersection of the $D_{25}\left(\sim 3.8 r_{e}\right)$ ellipse with the major axis. H1 extends towards the N-E corner of the field, where $\mathrm{H} 4(\sim 4.5 \sigma)$ can be observed. $\mathrm{H} 1$ is visible in the residual maps for both red and blue GC classes (Figure 8, upper right and lower left panels) with a strong indication of spatial segregation: red GCs in the $\mathrm{W}$ of the L structure and blue GCs in the E. H2 $(\sim 5.1 \sigma)$, slightly $\mathrm{E}$ of the intersection between the $D_{25}$ and the $\mathrm{S}$ end of the major axis, is more prominent in the highluminosity and blue GCs (Figure 8 lower right and left panels). $\mathrm{H} 3(\sim 4.6 \sigma)$ is located $\mathrm{E}$ of $\mathrm{H} 2$.

Both $\mathrm{H} 3$ and $\mathrm{H} 2$ lie along the $\mathrm{S}-\mathrm{W}$ direction, where the companion galaxy NGC4341 is located. Bogdán et al. (2012) reported that the centers of NGC4365 and NGC4342 are connected by a stellar-light stream. This stream is an indication of tidal interaction between NGC4365 and NGC4342 and corresponds to a stream of GCs detected by Blom et al. (2014) with kinematics consistent with the NGC4342 GC system (see Figures 10 and 11 from Blom et al. 2014). Using data from Blom et al. (2014), we find a marginally significant $(\sim 2.2 \sigma)$ GC overdensity consistent with the GC. H2 and H3 are roughly located at the $\mathrm{E}$ end of the GC stream. However, only one GC of the sample of 31 selected to have line-of-sight velocities compatible with the motion of the NGC4342 GC system (Figure 10 in Blom et al. 2014) is located within the region occupied by $\mathrm{H} 2$. The average line-of-sight velocity of the spectroscopic GCs from the list presented by Pota et al. (2013) located within H2 and $\mathrm{H} 3$ is compatible with the systemic velocity of the GC system of NGC4365. H5 $(\sim 4.1 \sigma), \mathrm{N}$ of the center of the galaxy, across the $D_{25}$ ellipse is also visible (with varying significance) in the other GC color and luminosity classes.

\section{9. $N G C 4621$}

Similar to NGC4526, the spatial distribution of GCs in NGC4261 (Figure 9, upper mid panel) shows two large and strong structures along the major axis. I1 $(>12 \sigma)$, in the $\mathrm{S}$ corner of the field observed, has similar significance and shape in the color and low-L classes (Figure 9. upper right and lower panels), but it appears positionally shifted in the high-L GCs (Figure 9, lower mid panel), I2 is similarly significant in all the classes, but its shape and size change considerably. For example, in the red (Figure 9, upper right panel) and high-L GCs (Figure 9. lower mid panel), the section of I2 that extends from the major axis towards N-E is barely visible, while in the blue (Figure 9, lower left panel) and the low-L GCs (Figure 9 lower right panel), it splits in three distinct components 

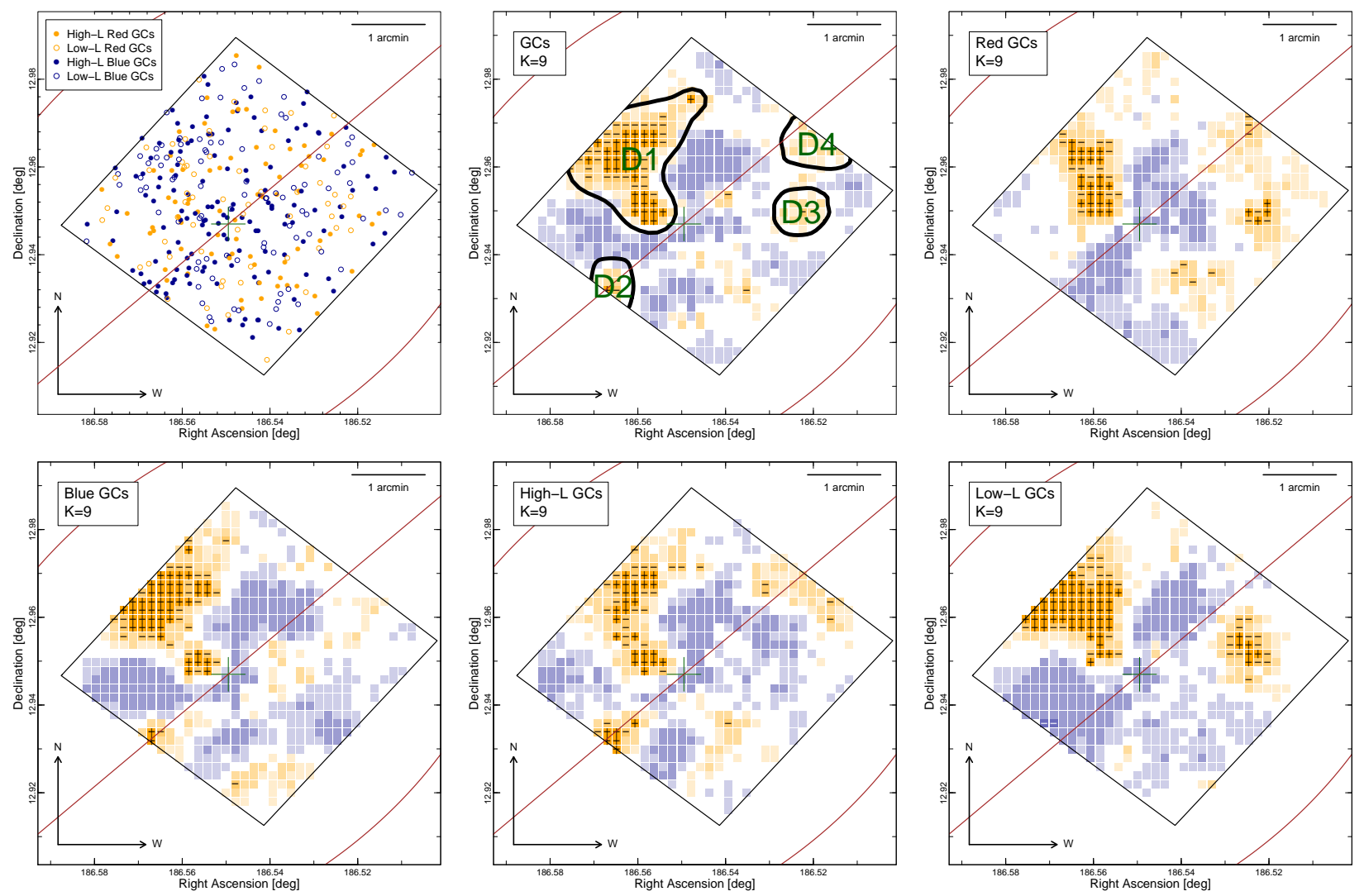

FIG. 4. - Scatterplot of the position of the GCs in NGC4406 and residual maps obtained for $K=9$ for the whole sample of GCs and the two color and luminosity GC classes. Refer to Figure 1 for a description of each panel.

that occupy most of the area of the HST field $\mathrm{N}$ of the center of the galaxy.

NGC4621 has large ellipticity (E4 Ferrarese et al. 2006), and displays a small stellar disk extending $\sim 10^{\prime \prime}$ from the center along the major axis. Its dust content is negligible (Ferrarese et al. 2006; di Serego Alighieri et al. 2013). In order to estimate the influence of the projection of an elongated three-dimensional distribution of GCs on the detection of the GC structures I1 and I2, we have used the same approach described in Section 4.7. The worst case scenario significance of the structure $\overline{11}$ is $\sim 5.8 \sigma$, while I 2 disappears as a single structure when the fraction of simulated GCs belonging to the component aligned with the major axis or the galaxy becomes larger than $55 \%$. Based on these results, we can assume that I1 is not entirely produced by projection effects while the real nature of 12 can only be determined by a more detailed modeling of the GC system, which is not possible with the data currently available.

\subsection{NGC4552}

NGC4552 (upper-mid panel of Figure 10) displays two large structures in the $\mathrm{W}$ side of the field. L1 $(\sim 8.7 \sigma)$, located in the S-W corner, extends parallel to the $D_{25}(\sim$ $3.6 r_{e}$ ) ellipse for $\sim 0.9^{\prime}$ and is prominent in blue and highL GCs. L2 $(\sim 7.3 \sigma)$, slightly $\mathrm{S}$ of the N-W corner of the field, within $D_{25}$, is clearly visible in all classes, except for the high-L GCs (Figure 10, lower mid panel). Two less significant structures L3 $(\sim 6.4 \sigma)$ and L4 $(\sim 5.7 \sigma)$ (visible in all classes of GCs), indicate the presence of a significant over-density close to the center of the galaxy. The fifth most significant residual structure L5 $(\sim 4.8 \sigma)$ in the W half of the galaxy at galactocentric distance $\sim 1^{\prime}$, suggests the existence of a long, spatially coherent elongated sequence of structures extending from the N-W corner of the field to the center, possibly encompassing also L2, L3 and L4.

Ferrarese et al. (2006) report the presence of two thin dust filaments extending $\sim 6^{\prime \prime}$ from the center towards N-NW direction and of patches of dust within a radial distance of $11^{\prime \prime}$. While these dusty regions could influence the reconstruction of the spatial distribution of GCs around the positions of these structures, their size is too small to affect them significantly.

\section{DISCUSSION}

The results presented in this paper confirm that localized spatial structures in the 2D distributions of $\mathrm{GC}$ systems are common. In the ten brightest Virgo cluster elliptical galaxies we have found GC over-density structures with a range of amplitudes, sizes, and shapes. We measure linear dimensions ranging from $\sim 1$ to $\sim 20 \mathrm{kpc}$ and azimuthal sizes ranging from $\sim 10^{\circ}$ to $\sim 90^{\circ}$. We observe GC structures with both simple, roughly circular shapes, and more complex forms. Below we discuss the characteristics of these structures, within the constraints provided by the existing limited observational coverage, and in the context of accretion of satellite galaxies (e.g. Bonfini et al. 2012, D'Abrusco et al. 2013, 2014a b). We use these results to set simple observation-based constraints to the size of the accreted satellite galaxies, that may have originated these structures.

\subsection{Location and shapes of GC over-density structures}



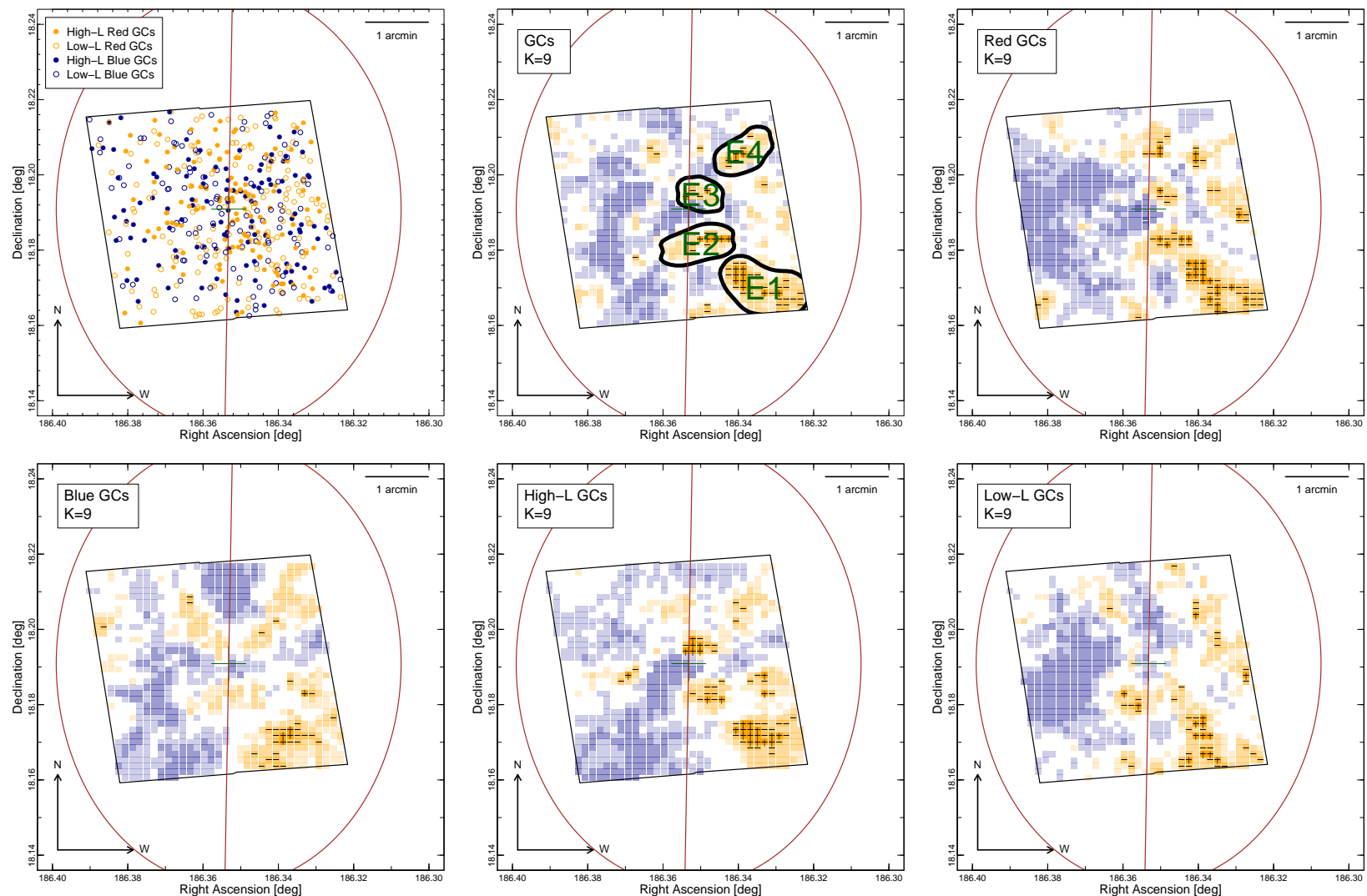

Fig. 5. - Scatterplot of the position of the GCs in NGC4382 and residual maps obtained for $K=9$ for the whole sample of GCs and the two color and luminosity GC classes. Refer to Figure 1 for a description of each panel.

Most of the over-density structures are located between $\sim 1^{\prime}$ and $2^{\prime}\left(0.2-0.8 r_{e}\right)$ from the center of the host galaxy where all the galaxies in our sample are reasonably well covered by the ACSVCS (Figures 11, 12). While the decline in the number of structures detected at smaller galactocentric radii may be real, we cannot exclude the effects of either incompleteness of the GC catalog in the central area of the galaxies, or the decreased ability of our method to select structures in compact regions with very high GC density. The decrease in the frequency of structures for radii larger than $2^{\prime}$ is definitely caused by the decrease of the galactic area covered by the ACSVCS observations. For $r>2.5^{\prime}$ only NGC4472, NGC4649 and NGC4365 have adequate HST coverage. Figure 12 illustrates the large scatter in the fraction of areas covered for the different galaxies in our sample (at $1 r_{e} \mathrm{~S}$ the fractional coverage varies between $0 \%$ and $100 \%$ per galaxy, with $\sim 35 \%$ of the total area covered) and the lack of homogeneous coverage for galactocentric radii larger than $0.5 r_{e}$.

In Figure 11 we show the spatial distribution of the over-density structures summarized in Table 3 . The significance-weighted central coordinates of the structures are plotted in the plane generated by their galactocentric radius, expressed as projected angular distances and in units of the effective radii of each galaxy, and azimuthal angle, measured counter-clockwise from the $\mathrm{N}$ direction of the major axis of the galaxy. We also show the areas of the galactocentric radius vs azimuthal angle plane that can be accessed with the GCs samples used in this paper as shaded colored background.
From Figure 11, it is evident that within the annulus $\sim 1^{\prime}-2^{\prime}$ where coverage is good for most galaxies, the smaller structures appear randomly azimuthally distributed in the $\left[0^{\circ}, 360^{\circ}\right]$ interval, suggesting that some of them might be the result of stochastic fluctuations, as discussed in Section 4. The medium and large structures (the largest size points in Figure 11) cluster along the major and minor axes $\left(\theta_{N} \leq 30^{\circ}\right.$ and $\theta_{N} \geq 330^{\circ}$ or $\left.150^{\circ} \leq \theta_{N} \leq 210^{\circ}\right)$. We explore this effect further in Figure 13 , where we plot the medium and large structures (see Section 4 and Table 4) in the galactocentric radius vs azimuthal angle plane.

The most extended large structure along the radial di-

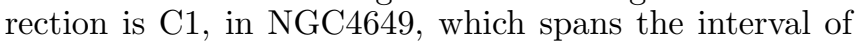
galactocentric distances $[0.5,2.8] r_{e}$. Other large structures that extend mostly along the radial direction are I1 and I2, in NGC4621, from $0.2 r_{e}$ to $1.1 r_{e}$ and from 0.1 $r_{e}$ to $1.2 r_{e}$ respectively, and G1 and G2 in NGC4526. All these structures are located along the major axis of their host galaxy in the whole range of radial distances covered by the ACSVCS observations. The distribution in angular position $\theta_{N}$ of the "large" structures (Figure 13 shows that 9 out of 14 of them are located near the major axis of the host galaxies. When the areas of the structures are considered, the larger structures are preferentially located along the major axes of the host galaxies, accounting for $\sim 86 \%$ of the total "structure area". This results appears consistent with the conclusion of Wang et al. (2013), reporting preferential alignment of GC system with the major axis in Virgo galaxies. However, 5 large structures (B1, D1, F1, L1, L2) follow 

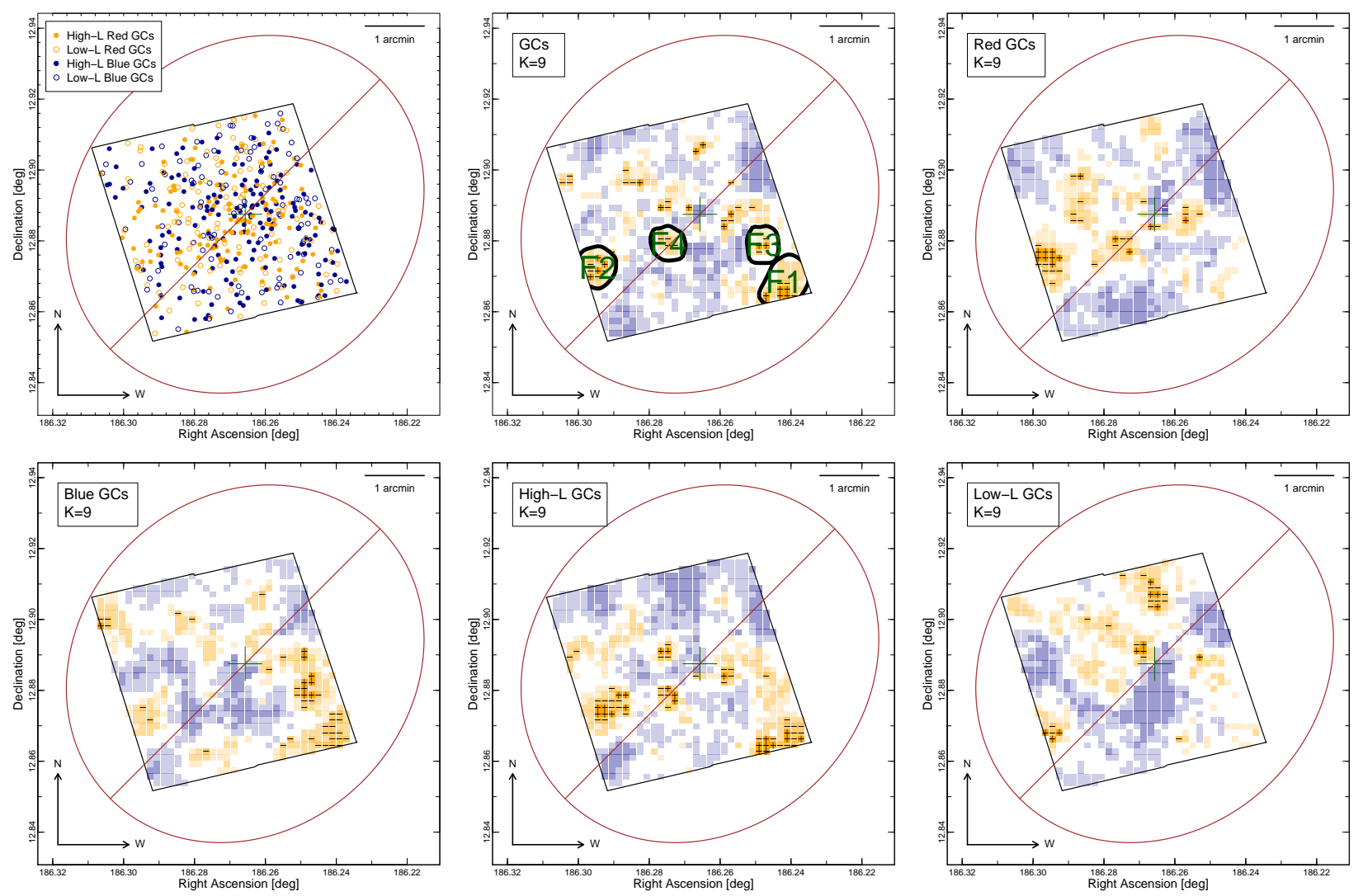

FIG. 6. - Scatterplot of the position of the GCs in NGC4374 and residual maps obtained for $K=9$ for the whole sample of GCs and the two color and luminosity GC classes. Refer to Figure 1 for a description of each panel.

the minor axis. This minor axis effect was not previously reported. It will need to be considered when trying to explain these results with theoretical simulations. The morphology of the host galaxy may play a role in these preferential orientations: the GC structures G1, G2, I1 and I2 observed in the galaxies NGC4526 and NGC4261 (classified as S0_3_(6) and E4, respectively, by Ferrarese et al. 2006) are almost perfectly aligned along the major axis of their galaxies.

The large coherent structures may be suggestive of the disruption and merging of a sizable companion galaxy. For at least NGC4649, stellar kinematics suggest a major dry (dissipationless) merger, with a lenticular galaxy as progenitor (Arnold et al.2014). However, we also note that the large structures may be slightly more prominent for the red GC subsystems. These large structures are on average 1.6 times more significant in the red GCs residual maps than the medium structures $(\sim 3.5 \sigma$ /pixel for large structures to $\sim 2.2 \sigma$ /pixel for medium structures), suggesting some dissipative merging-related GC formation (e.g., Bekki et al. 2002).

\subsection{Observational evidence of the assembly history}

For all the galaxies in our sample with the exception of NGC4374, NGC4526 and NGC4621, evidence of past merging or accretion of companions has been reported in the literature.

NGC4472 shows a fairly regular diffuse stellar distribution with elliptical isophotes (Ferrarese et al. 2006) within few $r_{e}$, but it has been long known to be interacting with its companion dwarf galaxy UGC7636 (McNamara et al. 1994). Its active accretion history has been confirmed by Janowiecki et al. (2010), which have observed a complex, extended shell system in the diffuse stellar light, suggesting radial accretion of a companion galaxy.

NGC4486 has regular isophotes but the kinematics of its GC system indicates that it is still accreting massive companions (Romanowsky et al. 2012); further evidence of stripping of low-luminosity dwarts along radial orbits come from the structures in its diffuse stellar light at large galactocentric radii (Janowiecki et al. 2010).

NGC4649 does not show significant structures in its stellar light distribution which might be associated to the GC over-density structure C1 (D'Abrusco et al. 2014a). However, kinematic evidence based on the Planetary Nebulae (PN) and GC systems, suggests that a merger has contributed to the assembly of this galaxy (Teodorescu et al. 2011, Das et al. 2011; Coccato et al. 2013). In particular, Das et al. (2011) showed that PNs and GCs might belong to separate dynamical systems at radii larger than $12 \mathrm{kpc}$. Arnold et al. (2014) used kinematics of the diffuse stellar light to discover disk-like outer rotation that might have originated from a massive lenticular galaxy progenitor.

NGC4406 also has small-scale streamers that can be explained by stripping of dwarf companions (Janowiecki et al. 2010); furthermore, observations of the cold dust emission in the surrounding area has confirmed that NGC4406 has stripped materials from the nearby spiral galaxy NGC4438 by tidal interaction (Gomez et al. 2010).

NGC4382 has boxy isophotes (Ferrarese et al. 2006) 

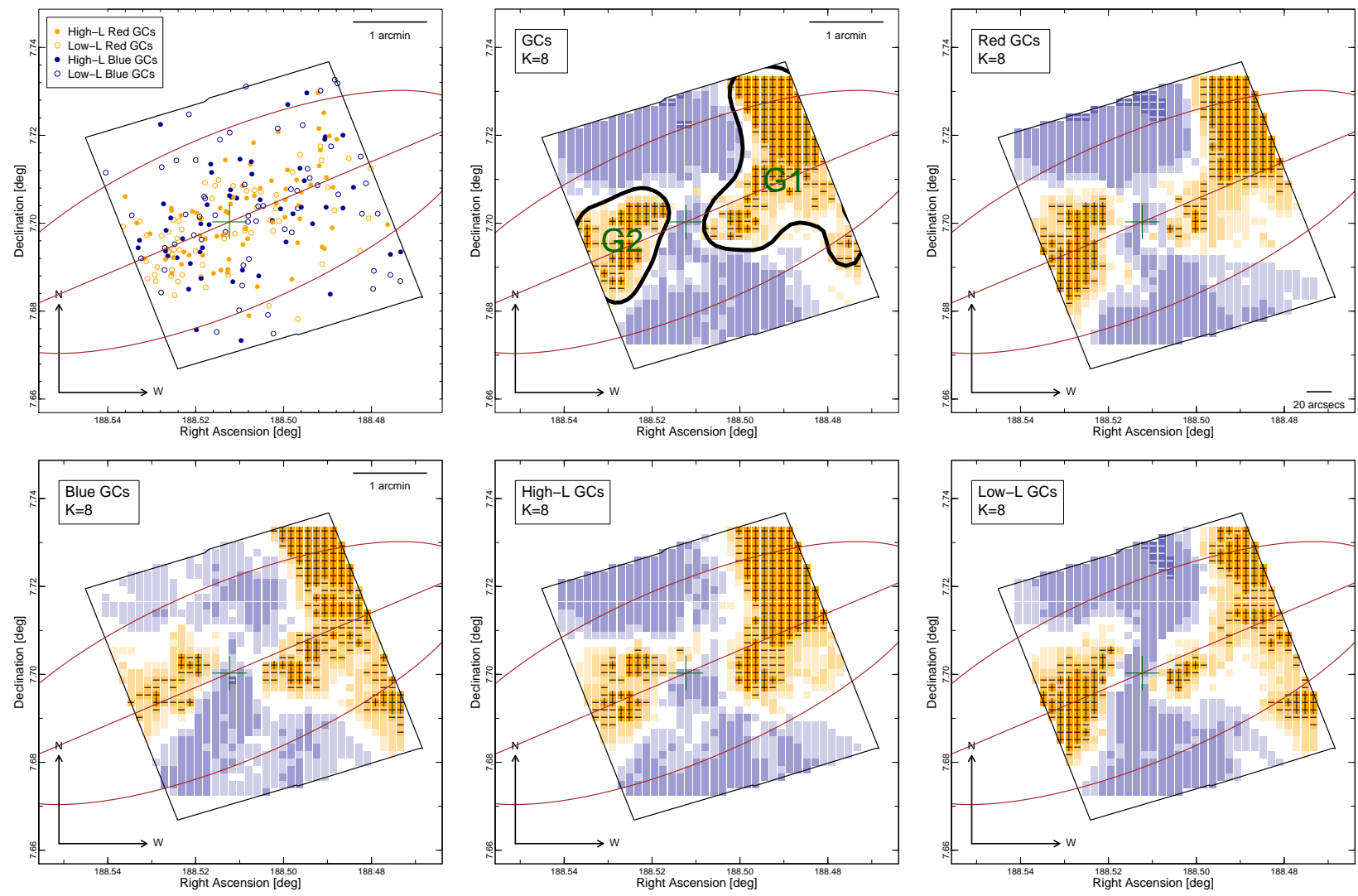

FIG. 7.- Scatterplot of the position of the GCs in NGC4526 and residual maps obtained for $K=9$ for the whole sample of GCs and the two color and luminosity GC classes. Refer to Figure 1 for a description of each panel.

and presents abundant fine structure in its diffuse stellar light (Schweizer et al. 1990), suggesting that it has undergone a major merger in the past 4-7 Gyr.

NGC4374, whose optical isophotes are very regular in the ACSVCS images (Ferrarese et al. 2006), shows a rapidly rotating central disk (Bower et al. 1998) but does not present evidence of kinematically decoupled components and it lacks significant tidal structures (Janowiecki et al. 2010). Arnold et al. (2014) found that the outer region of the galaxy show little to none rotation, in agreement with the expectations for a low rotator.

NGC4526 is a lenticular galaxy hosting a large dusty disk that extends along the major axis. Its isophotes become clearly boxy at $\sim 10^{\prime \prime}$ before becoming disky at $\sim 70^{\prime \prime}$ radius (Ferrarese et al. 2006).

NGC4365 is known for its kinematically decoupled core rotating along the major axis, which could be result of a major merger involving a spiral galaxy (Davies et al. 2001). This galaxy is in the process of stripping GCs from the companion NGC4342 (Blom et al. 2014).

NGC4621 shows a thin faint stellar disk in the ACSVCS images, but further evidence of a recent major merger or accretion event is missing.

NGC4552 has regular morphology in the diffuse stellar light within the $D_{25}$ (Ferrarese et al. 2006), but Janowiecki et al. (2010) detected a complex structure of shells and plumes that is suggestive of a complex accretion history involving either multiple small accretions or a major merger.

\subsection{GC structures in color and luminosity classes}

Color and luminosity differences in the GC overdensity structures are noticeable in several galaxies (Section 4. Table 4). While some of these differences may be due to statistical noise, some are significant and may pose constraints on the origin and evolution of the structures. Among the medium and large GC over-density structures, spatial segregation has been detected for red/blue GC classes in H1 (NGC4365), and for high-L/low-L GCs in A1 (NGC4472), C1 (NGC4649), E2 (NGC4382) and L5 (NGC4552). We selected the GC structures with spatial segregation by comparing the significances of $\mathrm{GC}$ structures in each of the color (red/blue) and luminosity (high-L/low-L) classes. The structures for which the difference of significance was larger than $3 \sigma$ were visually inspected to ascertain the presence of spatial segregation. In NGC4472, the luminosity segregation in the large structures A1 suggests an incoming tangential GC stream that then turns into the radial direction. In this stream, the low-luminosity GCs are prevalent in the inner radial direction, while the more luminous GCs are mostly found in the section of the structure tangential to the $D_{25}$. This evidence suggests that dynamical friction (Fall \& Zhang 2001; Binney \& Tremaine 2008) can be ruled out as the only mechanism responsible for the observed segregation, as it would act more vigorously on the luminous, more massive clusters that should be observed at smaller galactocentric distances than the less massive, low-L GCs. Kinematic observations are needed to shed light on the origin of the spatially segregated high- and low-L components of A1. We stress that, lacking observational confirmation or a detailed explanation 

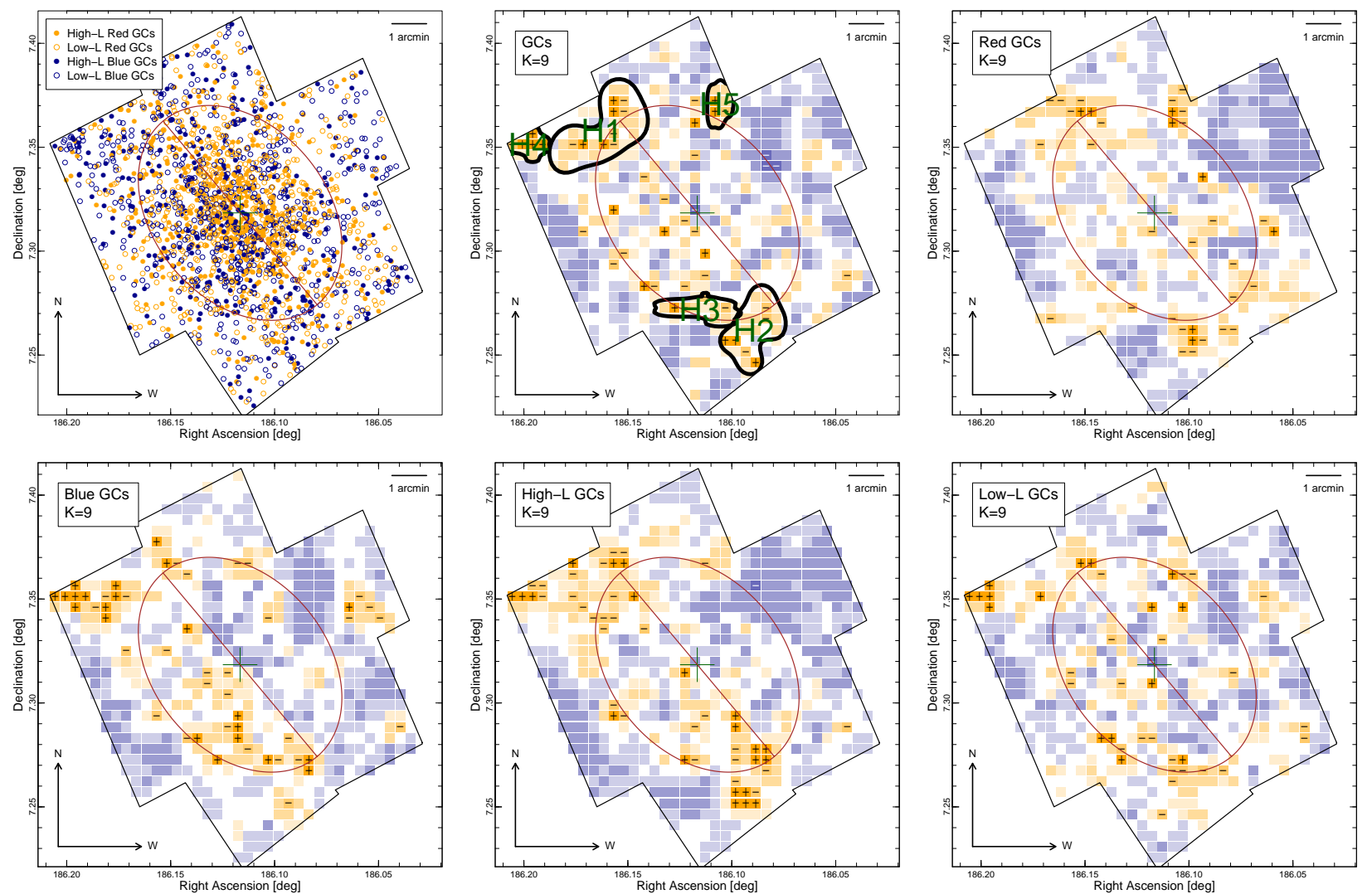

Fig. 8. - Scatterplot of the position of the GCs in NGC4365 and residual maps obtained for $K=9$ for the whole sample of GCs and the two color and luminosity GC classes. Refer to Figure 1 for a description of each panel.

of the physical mechanisms responsible for the observed properties of this GC structure, the two components in A1 could have originated in distinct events.

The spatial segregation of high-L/low-L GCs observed in $\mathrm{C} 1$ (NGC4649) could be tentatively explained as a result of differential dynamical evolution of the GC color classes formed during the major merger experienced by the host galaxy, as discussed by D'Abrusco et al. (2014a). Color differences in the distribution of the GC overdensity structures may be related to the prevalent GC population of merging satellite galaxies, if these are the results of dry mergers. Gas-rich mergers may increase the localized red GC population. We note that the highly incomplete spatial coverage of our sample (see Figure 12) does not allow us to assemble a full picture of either the shapes or the characteristics of these GC features.

\subsection{GC structures and local galaxy density}

Figure 14 shows the location of the ten galaxies studied in this paper in the region of the sky covered by the Virgo cluster. We compare their position with the local galaxy density, which we have evaluated from the cata$\log$ of confirmed and candidate Virgo galaxies of Binggeli et al. (1985). This catalog is complete to $B_{T} \sim 18 \mathrm{mag}$ and covers $\sim 140 \mathrm{deg}^{2}$. The large GC structures detected in each galaxy, color-coded based on their membership, are shown in the insets of Figure 14 for reference. We calculated the average fraction of the observed galaxy areas belonging to medium and large GC structures for both higher- and lower-density regions of the Virgo cluster using the data shown in Table 4 . Only 2.1\% of the total observed area of the three galaxies (NGC4406, NGC4486 and NGC4374) located within the inner green line in Figure 14, which is the isodensity contour corresponding to $50 \%$ of the peak density of the Virgo cluster galaxies, is occupied by large GC structures (16.9\% if both medium and large structures are considered). The fraction of the area of the remaining seven host galaxies located outside of the $50 \%$ isodensity contour which is occupied by large structures grows to $8.5 \%$, while it remains almost constant (16.6\%) if both medium and large GC structures are considered. While these numbers seem to indicate that galaxies in the highest-density regions of Virgo tend to have smaller GC structures than galaxies located elsewhere, we note that the uneven spatial coverage of ACSVCS is a source of uncertainty in these conclusions. For instance, if we consider only the five galaxies outside of the outer green line (the isodensity contour corresponding to the $33 \%$ of the peak density), the fractions of area occupied by large and large plus medium GC structures become $2.8 \%$ and $15.2 \%$, respectively. This inversion of the trend, though, is likely caused by the incomplete spatial coverage of the sample: NGC4649 and NGC4365, the only two galaxies almost completely observed within the $D_{25}$, are both located outside of the $33 \%$ contour, and their presence "dilutes" the average fractional area occupied by large structures. Another possible source of bias is the uneven distribution of galaxy morphologies in higher- and lower-density regions: lenticulars and ellipticals with significant ellipticity are mostly located in the lower-density regions, while two of the three galaxies within the $50 \%$ isodensity con- 

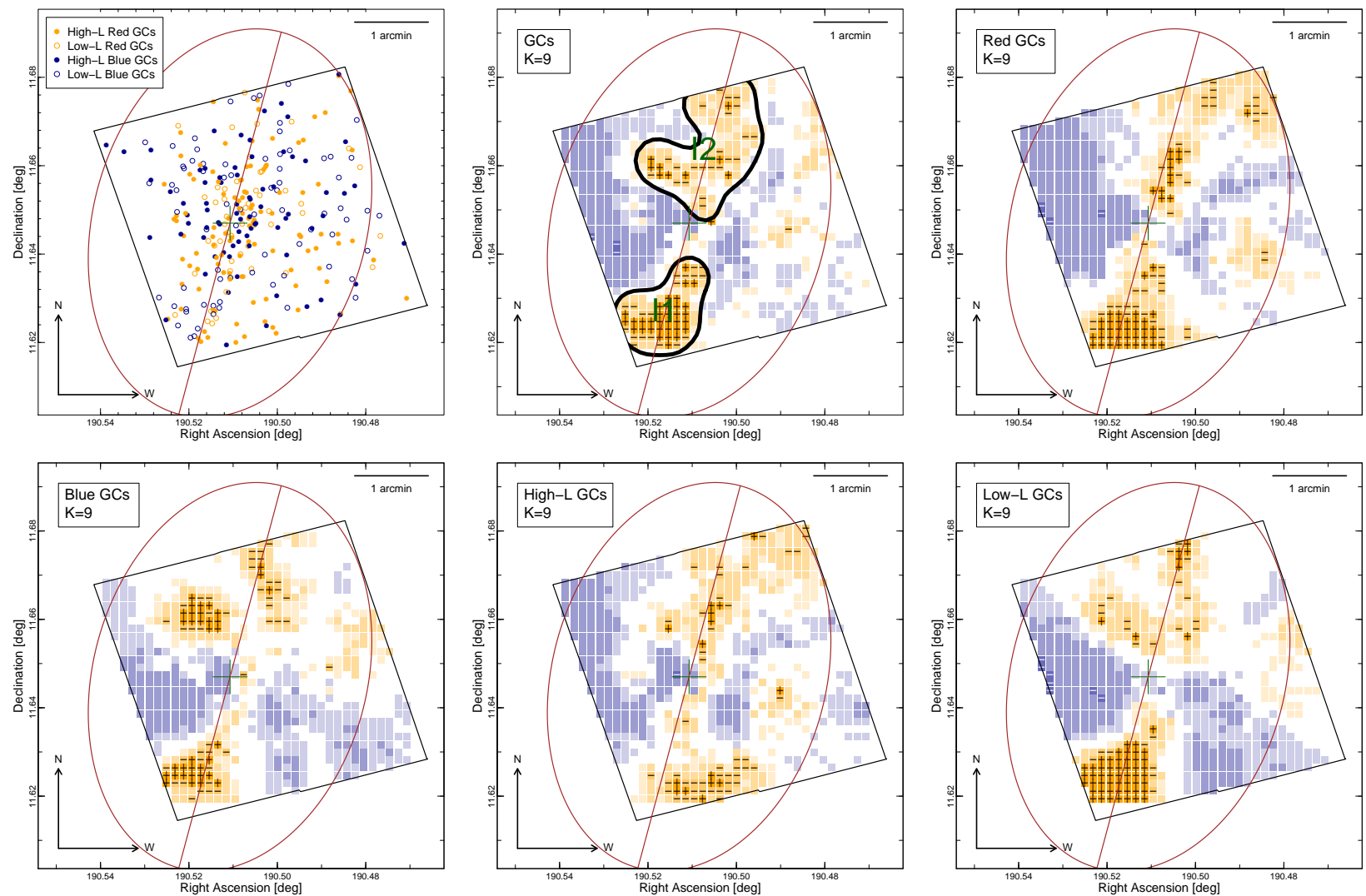

FIG. 9.- Scatterplot of the position of the GCs in NGC4621 and residual maps obtained for $K=9$ for the whole sample of GCs and the two color and luminosity GC classes. Refer to Figure 1 for a description of each panel.

tour (NGC4486 and NGC4374) show negligible to small ellipticity (E0 and E1, respectively).

As discussed in Section 5.1, the large GC structures are on average more significant $(\sim 1.6$ times $)$ in the red GCs than in the general GC population. When split according to the environment density and normalized by the area of the structures, the large GC structures are even more significant in the residual maps generated by red GCs in galaxies in low-density regions than the large structures in medium and high-density areas of the cluster. In particular, the large GCs structures associated to host galaxies outside of the $33 \%$ of the peak density region are, on average, 5.3 times more significant than GC structures in all GCs, while the same excess probability decreases to 3.5 and 1.2 for large structures in the medium and high-density regions.

Our findings are in agreement with two different recent accretion histories for the galaxies in the higherand lower-density regions of the cluster. As suggested by hydrodynamical simulations (Millennium simulation, see Springel et al. 2005) and observations of the local clusters of galaxies (for instance, for Coma cluster Smith et al. 2012), major merging events occurred earlier in the high-density regions at the center of the clusters. For instance, according to the Millennium simulation, an average galaxy currently observed at small clustercentric distance joined a halo whose size is typical of the Virgo cluster 8.9 Gyr ago, corresponding to redshift $z=1.3$. At a distance of $2 \mathrm{Mpc}$, the same galaxy reached a clusterlike environment only $4.8 \mathrm{Gyr}$ ago $(z=0.5)$ (Smith et al. 2012). Observationally, mergers in the distant past have depleted of similar-sized companion the environment of the largest galaxies, which are currently observed in the deepest potentials. Therefore, only recent accretions of smaller-than-average galaxies (satellites) can be expected in the densest areas of clusters. Instead, major mergers (or wet mergers with larger mass ratios) may have occurred in the more recent past in lower density areas, producing the large scale, spatially extended GC structures mostly located along the major axis of the host galaxies, that we observe.

\subsection{Constraints on potential progenitors of the $G C$ structures}

The $\Lambda$ CDM model of hierarchical galaxy formation (White \& Rees 1978, Di Matteo et al. 2005) predicts continuous galaxy evolution via merging and accretion of satellites. Supporting evidence are provided by recent observations of the local Universe, including the Sagittarius Stream (Ibata et al. 1994); Milky Way companions undergoing tidal disruption (Belokurov et al. 2006), and streams and dwarf galaxies in the halo of M31 (McConnachie et al. 2009). The kinematics of the GC system of NGC4486 also suggest active accretion of satellite galaxies (Romanowsky et al. 2012), as do the presence of GC streams in a few ellipticals (e.g., Strader et al. 2011, Blom et al. 2012a). Other results (Bonfini et al. 2012) and our previous work on the 2D GC distributions of E galaxies (D'Abrusco et al. 2013, 2014a b) as well as this work suggest that GC systems may bear the imprint of these effects, as fossil remnants of accretion events of small dwarf galaxies (Peñarrubia et al. 2009).

Based on our results, we assume that the structures we 

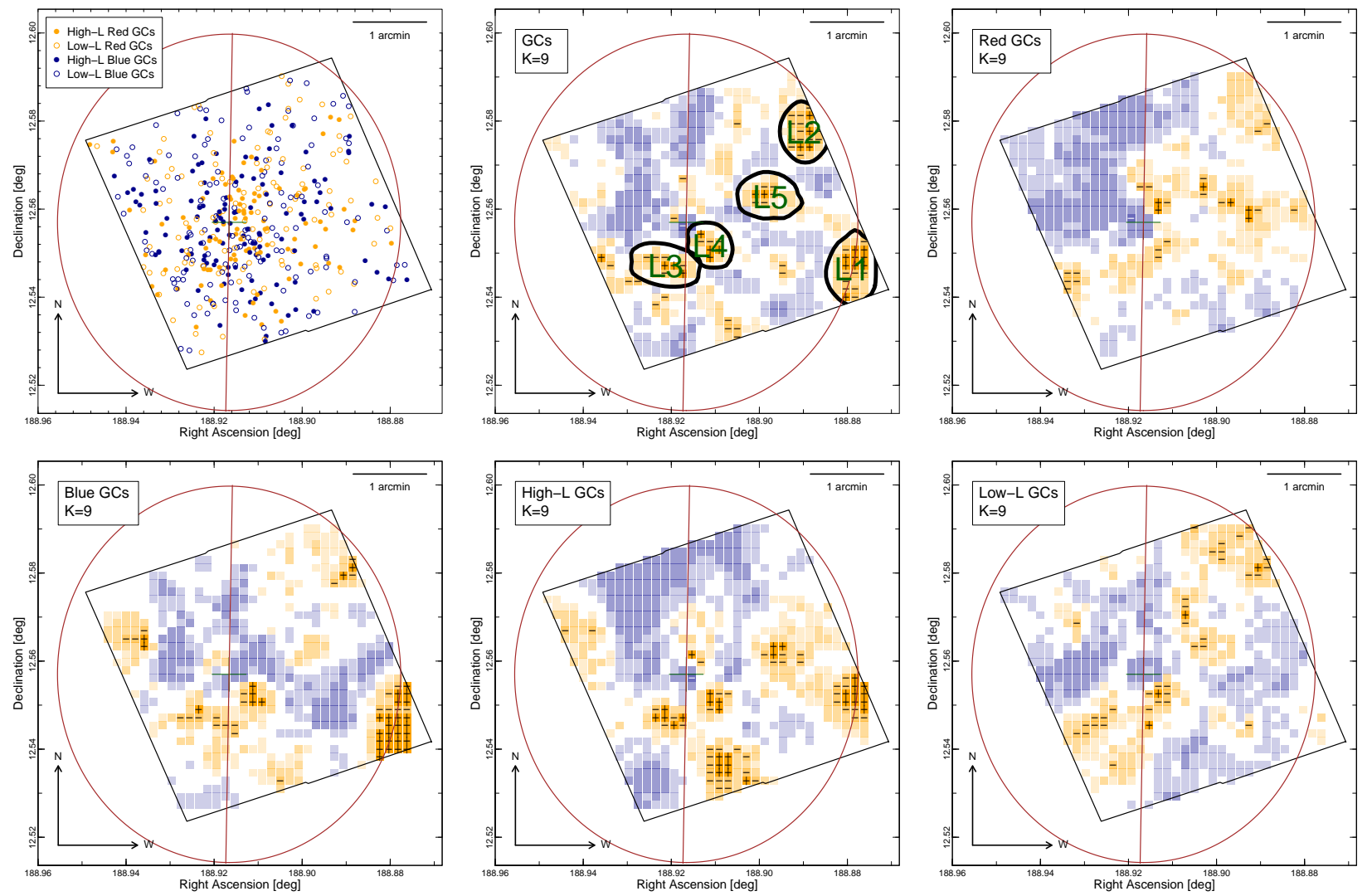

FIG. 10. - Scatterplot of the position of the GCs in NGC4552 and residual maps obtained for $K=9$ for the whole sample of GCs and the two color and luminosity GC classes. Refer to Figure 1 for a description of each panel.

have detected in the 2D GC distributions of the ten most luminous Virgo ellipticals are the remnants of accreted galaxies, detected over the smooth, relaxed GC distribution of the main galaxy. A discussion of the caveats to this assumption can be found in Section 5.6. Using the excess number of GCs in the detected over-density structures, we can set lower limits on the masses of their parent galaxies, by using the relations of Harris et al. (2013) between the number of GCs and the dynamical mass of elliptical galaxies. We stress that these estimates are lower limits, because GCs may be destroyed or reduced in these encounters (see Fall \& Zhang 2001, for an extensive review on the dynamical evolution of GCs). Moreover, detailed numerical simulations of the evolution of GCs in these scenarios are lacking.

We considered all the GC medium and large overdensity structures of the ten Virgo cluster galaxies in our sample (Table 5). Among these structures, we will focus, in particular, on the over-density structures that are clearly identifiable also in the residual maps obtained from the blue GCs, because metal-poor GCs could have been either produced early in the assembly history of the host galaxy or subsequently added through accretion of low-mass, metal-poor satellite galaxies (Brodie \& Strader 2006 Peng et al. 2008). These structures are A1, A3, B1, B2, B3, B4, B5, D2, E1,E2, E4, F1, F2, F3, G1, G2, H1, H2, H3, H4, H5, L1, L2, L3, L4 (the other structures for which the satellite properties have been calculated for comparison only are marked with an asterisk in Table 5).

To estimate the number of GCs in each over-density structure, we used both the observed radial density pro- file $N_{\text {exc,GC }}$ (which is a measure of the azimuthally averaged radial GC distribution in a galaxy) and the minimal radial density profile $N_{\text {exc,GC }}^{(\mathrm{MRD}}$, obtained by using the minimum value for each given azimuth (Table 5). These two different definitions of the density radial profiles provide reasonable estimates of the minimum and maximum size of the GC systems in the structures, and were both used to evaluate the range of possible values of the basic properties of the accreted galaxies. We then corrected these estimates for incompleteness using the GC luminosity functions determined with the ACSVCS survey data for Virgo cluster galaxies by Jordán et al. (2007). The average additive corrections are $\sim 0.6$ and $\sim 0.9$ GCs per density structure for the two radial density profiles used, respectively. We then applied the relations $N_{\mathrm{GCs}}=M_{\mathrm{dyn}}^{1.04 \pm 0.03}$ and $N_{\mathrm{GCs}}=\left(r_{e} \sigma_{e}\right)^{1.29 \pm 0.03}($ Harris et al. 2013), where $M_{\mathrm{dyn}}$ is the dynamical mass (Wolf et al. 2010) defined as:

$$
M_{\mathrm{dyn}}=\frac{4 r_{e} \sigma_{e}^{2}}{G}
$$

which approximates the total baryonic mass of the galactic bulge within the effective radius $r_{e}$, where the contribution of the dark-matter halo is negligible (Graves

4 The minimal radial density (MRD) profile is defined as the density profile obtained by setting the density in each radial bin equal to the lowest density observed in that radial bin. In physical terms, this assumption translates to assuming that all positive residuals in the residual map of GCs can be interpreted as accreted GC systems from distinct satellite galaxies. 

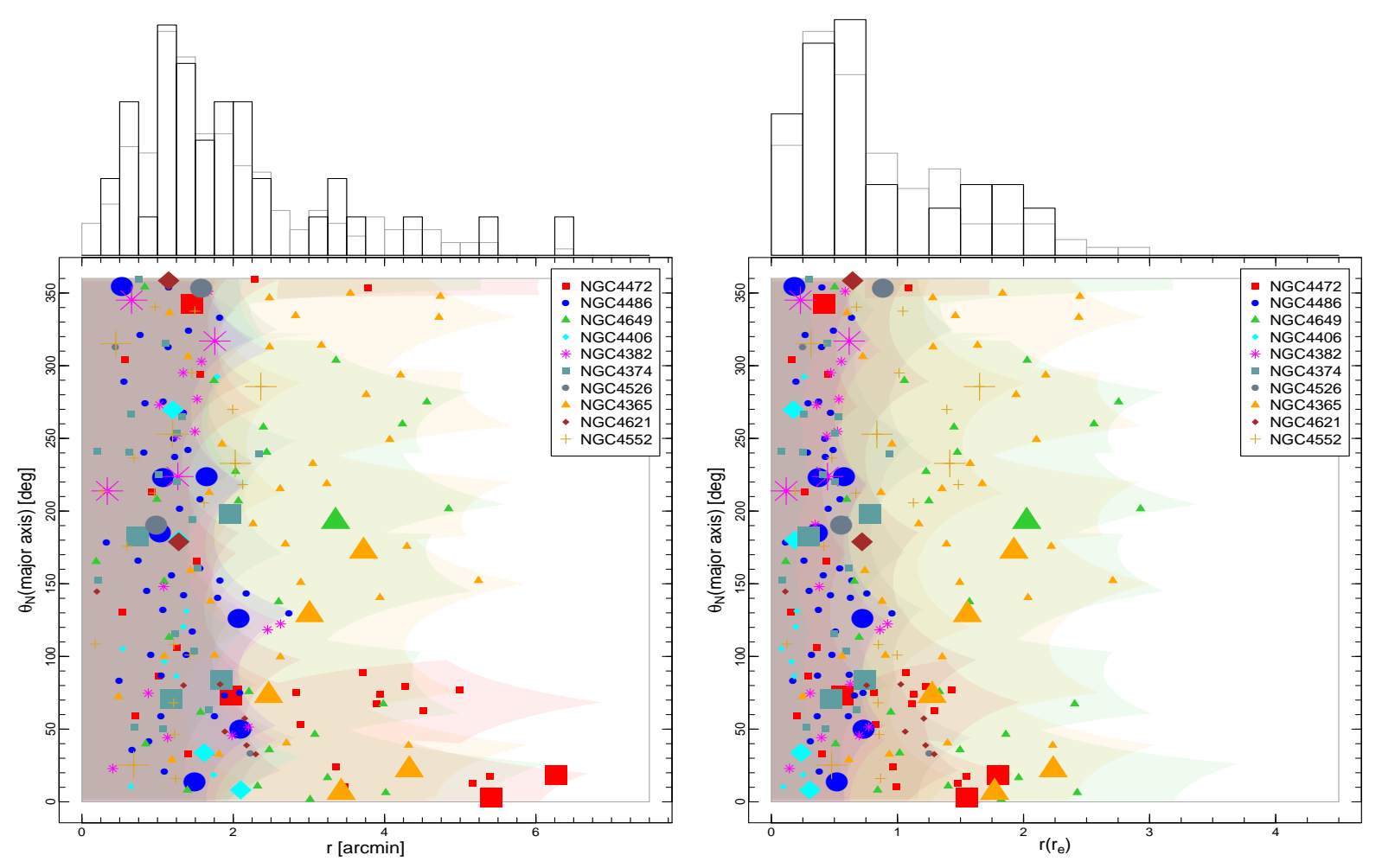

Fig. 11. - Distribution of the over-density structures shown in Table 3 in the galactocentric radius vs azimuthal angle plane (measured counter-clockwise from the $\mathrm{N}$ direction of the major axis of the galaxy). In the left panel, the galactocentric radius is expressed as projected angular distance, while in the right panel we use units of the effective radii $r_{e}$ of each galaxy as estimated by Ferrarese et al. (2006) (Table 1). In both panels, the shaded background regions represent the areas of the plots that can be accessed with the GC sample used in this paper for each galaxy. These regions follow the same color-coding used for the points. In both plots, the large points represent the significance-weighted central positions of the medium and large GCs structures discussed in Section 5.1 and the gray and black marginal histograms show respectively the normalized distributions of the galactocentric radii of all GCs structures (gray) and large only GCs structures only (black).

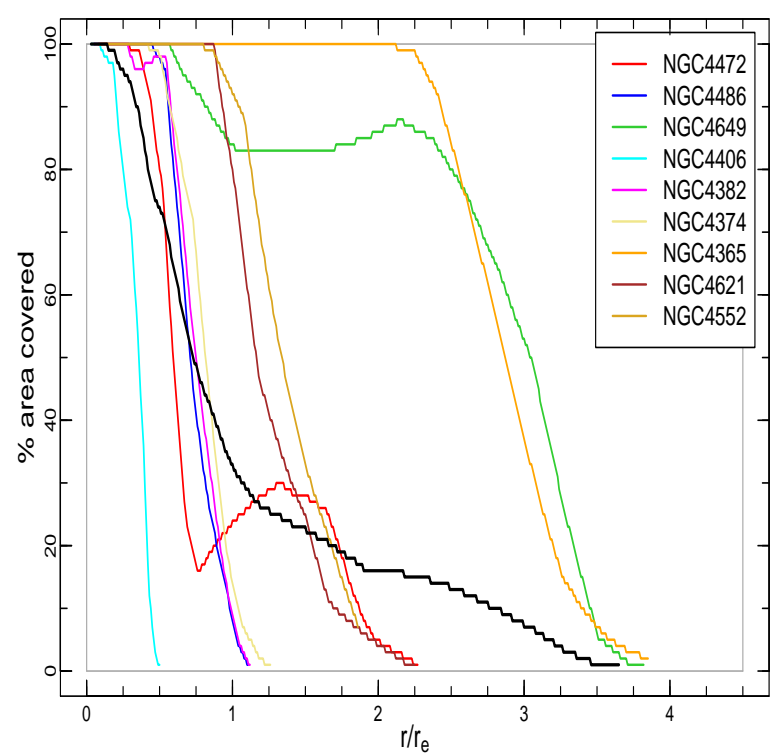

FIG. 12.- Fraction of the area of each galaxy as a function of the galactocentric distance in units of the effective radii $r_{e}$ covered by the footprints of the HST ACS observations used to extract the catalogs of GCs employed in this paper. The black line represents the fraction of total area covered by the observations for the entire sample of galaxies.

\& Faber 2010); $\sigma_{e}$ is the central velocity dispersion of the galaxy derived from spectroscopic observations of the bulge available in the literature (see Harris et al. 2013 . and references therein). Harris et al. (2013) found that $r_{e} \sigma_{e}$ strongly correlates with the size of the GC population.

The results (Table 5) show that under our assumptions, the average fraction of mass contributed by each accretion event (among the GC structures with no asterisk) is $\sim 1.3 \%$. The largest fraction of dynamical mass of the host galaxy accreted through one satellite is $6.9 \%$ (10.1\% for MRD profile) for G2 in NGC4526 (assuming that each GC structure corresponds to one accreted satellite). The largest fraction of total mass gained by the host galaxy through all the accretion events associated to the observed GC structures is $\sim 12.2 \%(\sim 17.8 \%$ for MRD profile) for NGC4526 (G1 and G2)). Among the other galaxies, the total fraction of dynamical mass contributed by all accretion events exceeds $4 \%$ only for NGC4365 for the observed density profile; two more galaxies exceed this limit when using the MRD profiles (NGC4365 and NGC4621). The estimated dynamical masses of the accreted satellite galaxies span the $[\sim 8.5, \sim 10.2]$ $\log M / M_{\odot}$ for the observed radial density profiles and [ 8.3, 10.3] $\log M / M_{\odot}$ for MRD profiles. Considering that these are lower limits, they are consistently larger that the typical masses of dwarf elliptical galaxies (dEs) $M \sim 10^{7} M_{\odot}$ (Mateo et al. 1993). Using the relation $N_{\mathrm{GCs}}=M_{\mathrm{dyn}}^{0.37 \pm 0.09}$ obtained from Harris et al. (2013) for dEs, the estimated masses of the accreted galaxies, on average, are only $\sim 20 \%$ smaller than the masses ob- 


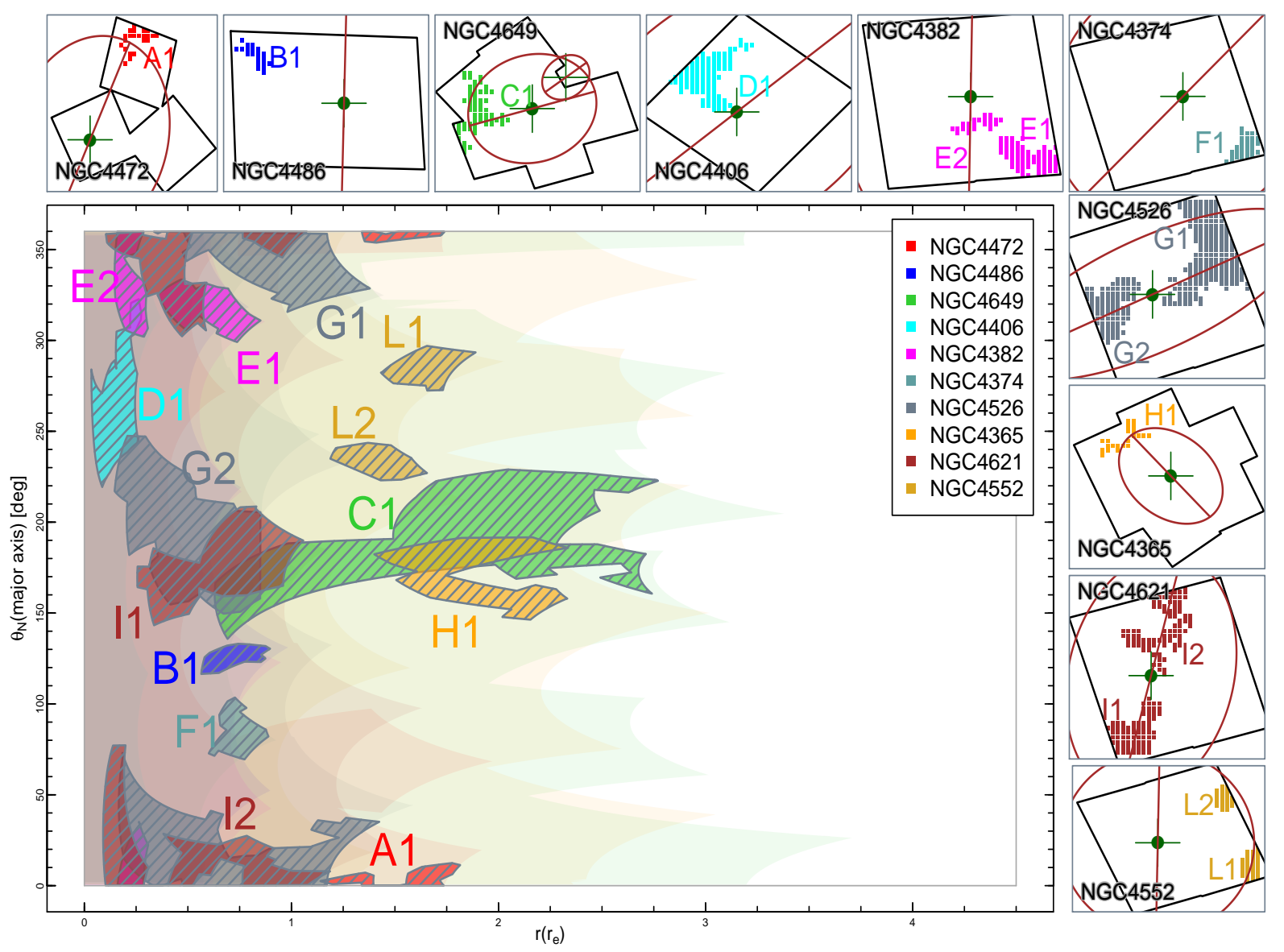

FIG. 13.- Boundaries of the "large" (more than 19 pixels) GC over-density structures in the galactocentric distance (expressed in units of the effective radii $r_{e}$ ) vs azimuthal angle plane. The plots in the margins show the "large" structures in the R.A. vs Dec. plane. The background regions are color-coded based on their membership to the galaxies of our sample.

tained with the relation for all elliptical galaxies, and still significantly more massive than typical dEs. This fact may represent another indication that these galaxies have undergone multiple mergers, which is a reasonable hypothesis since they are members of the Virgo clusters, and some of them even in sub-groups in Virgo (see Section 5.4.

Figure 15 compares the estimates of the distribution of dynamical masses of the satellites of the Virgo cluster galaxies (from Table 5), with the distributions of masses of the Milky Way (McMillan 2011) and M31 satellites (McConnachie 2012). While we have used for all the M31 satellites the estimates of the dynamical masses within the observed half-light radius available in (McConnachie 2012), different definitions have been used to determine the masses of the MW satellites, that usually yield mass estimates than can be systematically larger than the dynamical masses for the same systems. Even within the uncertainties and the possible systematic differences in the masses of the host galaxies, our estimates point to larger satellites in the Virgo galaxies. Considering the similar mass of the MW, M31 and the Virgo ellipticals, environment may play a role. As shown, the mass of each accreted objects is a small fraction of the total mass of the main galaxy. Therefore, one would expect only mild morphological and kinematic disturbances (assuming a dry merger) that would not be easily discernible from the light of the galaxy. Furthermore, one would expect a correlation between the mass of the accreted satellite and the number of excess GCs. However, such a correlation is easily diluted by multiple cannibalism events, the time since coalescence, and the geometry of the interaction. Simulations of the evolution of the GC populations for a period of several Gyr after a dry merger are needed to address these issues.

In Section 5.4. we reported that the GC structures observed in host galaxies located in lower-density regions of the Virgo cluster are more significant in the distribution of red GCs than in all GCs, than structures observed in galaxies located in higher-density regions. We argued that this evidence, in agreement with the predictions of cosmological simulation of the assembly history of galaxies in cluster, indicates that recent major mergers are mostly responsible for the properties of the GC spatial distribution in the outskirts of Virgo, while the innermost galaxies, recently, have only accreted smaller companion galaxies. The fraction of dynamical mass contributed to the host galaxy by a single progenitor (shown in Table 5) does not exceed $5 \%$ (10\% for MRD profiles) for the five galaxies (NGC4472, NGC4649, NGC4382, NGC4365 and NGC4621) located outside of the $33 \%$ isodensity contour shown in Figure 14. No single GC structure accounts for $25 \%$ of the mass of the final galaxy, which is the threshold used to label major mergers (i.e., mergers where the progenitors have 3:1 or lower mass ratio). Even assuming that all observed GC structures originate from a sin- 


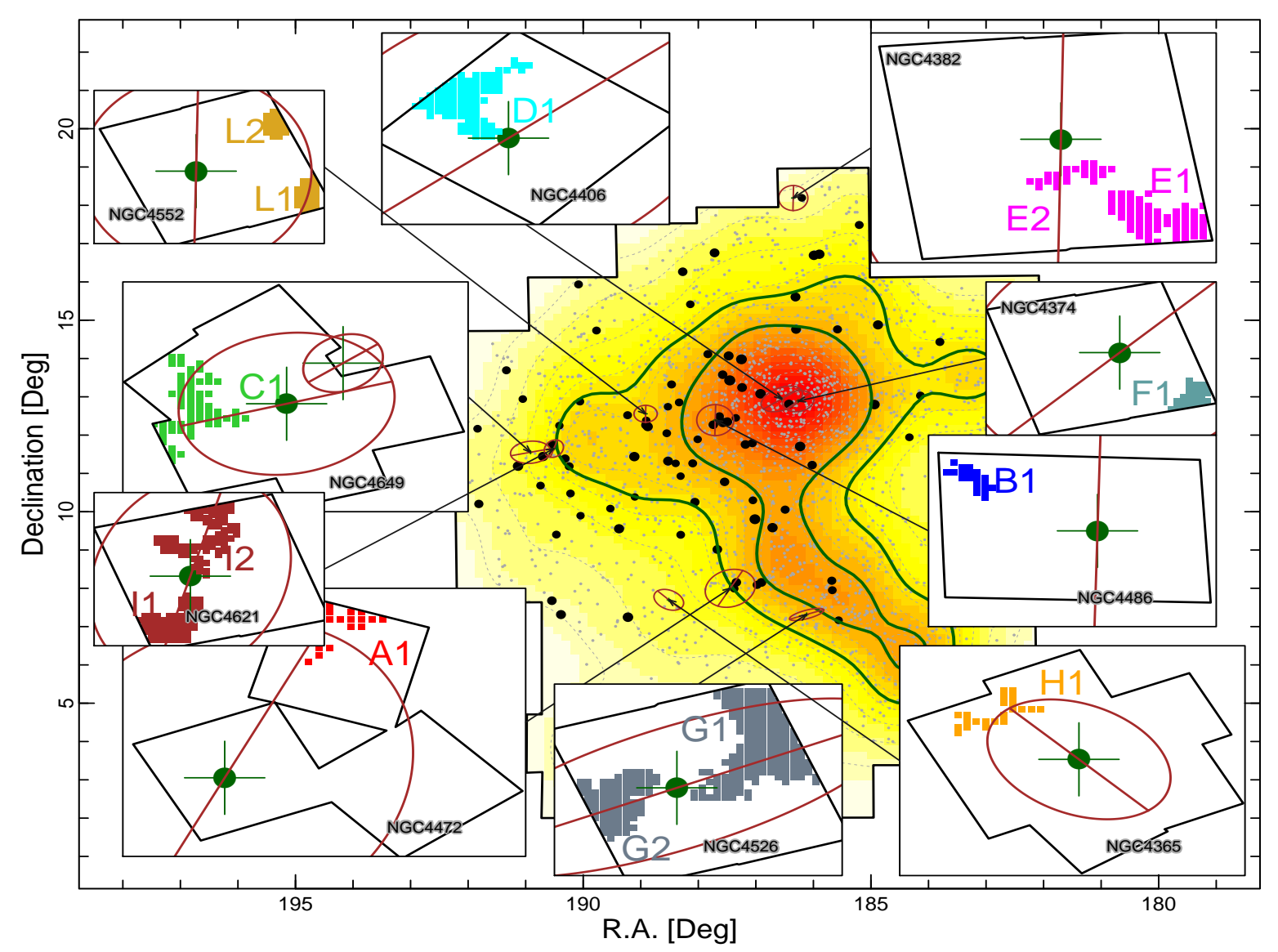

FIG. 14.- Positions of the ten Virgo cluster galaxies whose GC spatial distribution has been investigated in this paper (the $D_{25}$ isophotes of the galaxies, scaled for visibility, are also plotted). On the background, the surface density of confirmed and candidate Virgo cluster galaxies calculated from the Binggeli et al. (1985) catalog is shown. The two green lines are associated, from the center to the outskirts of the cluster, to the 50\% $\left(\sim 40\right.$ galaxies/deg $\left.{ }^{2}\right)$ and $33 \%\left(\sim 27\right.$ galaxies $\left./ \mathrm{deg}^{2}\right)$ fractions of the peak density. The insets display the location of the large GC structures within each host galaxy.

gle accretion event, the total fraction of host galaxy dynamical mass contributed by the progenitors (including also GC structures marked with asterisks in Table 5) for these five galaxies are $1.7 \%$ for $\mathrm{NGC} 4472,2.2 \%$ for NGC4649, 3.7\% for NGC4382, 5.9\% for NGC4365 and $4.6 \%$ for NGC4621 (with MRD profiles the fractions are $1.2 \%, 19.2 \%, 1.6 \%, 6.5 \%$ and $7.9 \%$ respectively). The disagreement between our discussion in Section 5.4 and these estimates depends mostly on two factor. Firstly, in our simple model that employs either the observed or MRD profiles to calculate the excess number of GCs for each GC structure, the dynamical evolution of the GCs is not taken into account. The changes in the mass function of GCs due to internal mechanism (stellar evolution and two-body relaxation) and to interactions with the host galaxy potential (gravitational shocks and dynamical friction; Fall \& Zhang 2001) all contribute to lower the estimated number of excess GCs and, based on the Harris et al. (2013) relations, to underestimate the dynamical mass of the progenitors. The second factor is represented by the fact that major and/or wet mergers are usually conducive of new star formation and, consequently, the formation of new GCs. The use of the relations derived by Harris et al. (2013) implies a "passive" scenario where GCs are donated from the progenitors to the main galaxy; it does not take into account the effect of the merger on the GC system of the resulting galaxy. Moreover, the uncertainties on the GC specific frequencies for galaxies of large luminosity and different morphology that are typically involved in wet/major mergers and that can affect the scatter and accuracy of the $N_{G C}-M_{\text {dyn }}$ relation (see discussion in Harris et al. 2013), further undermines the validity of the estimated dynamical masses in the massive tail of the mass distribution of the progenitors. It is also worth mentioning that major mergers are not the only possible way of forming new GCs, as wet mergers with mass ratios larger than 3:1 may also produce significant amounts of star formation and, in turn, new young, metal-rich GCs.

Detailed simulations of the formation and evolution of the GC populations in mergers of different types would be crucial to reconcile the tension between the assembly histories discussed in Section 5.4 and the inferences about the progenitor galaxies made in this Section, and would contribute to paint a more realistic and complex views of the evolution of GCs and their host galaxies in environment like the Virgo cluster.

\subsection{Caveats}

Our inferences on the properties of the progenitors of the GC structures (Section 5.5) are based on the hypothesis that all the structures originated by either accretion 


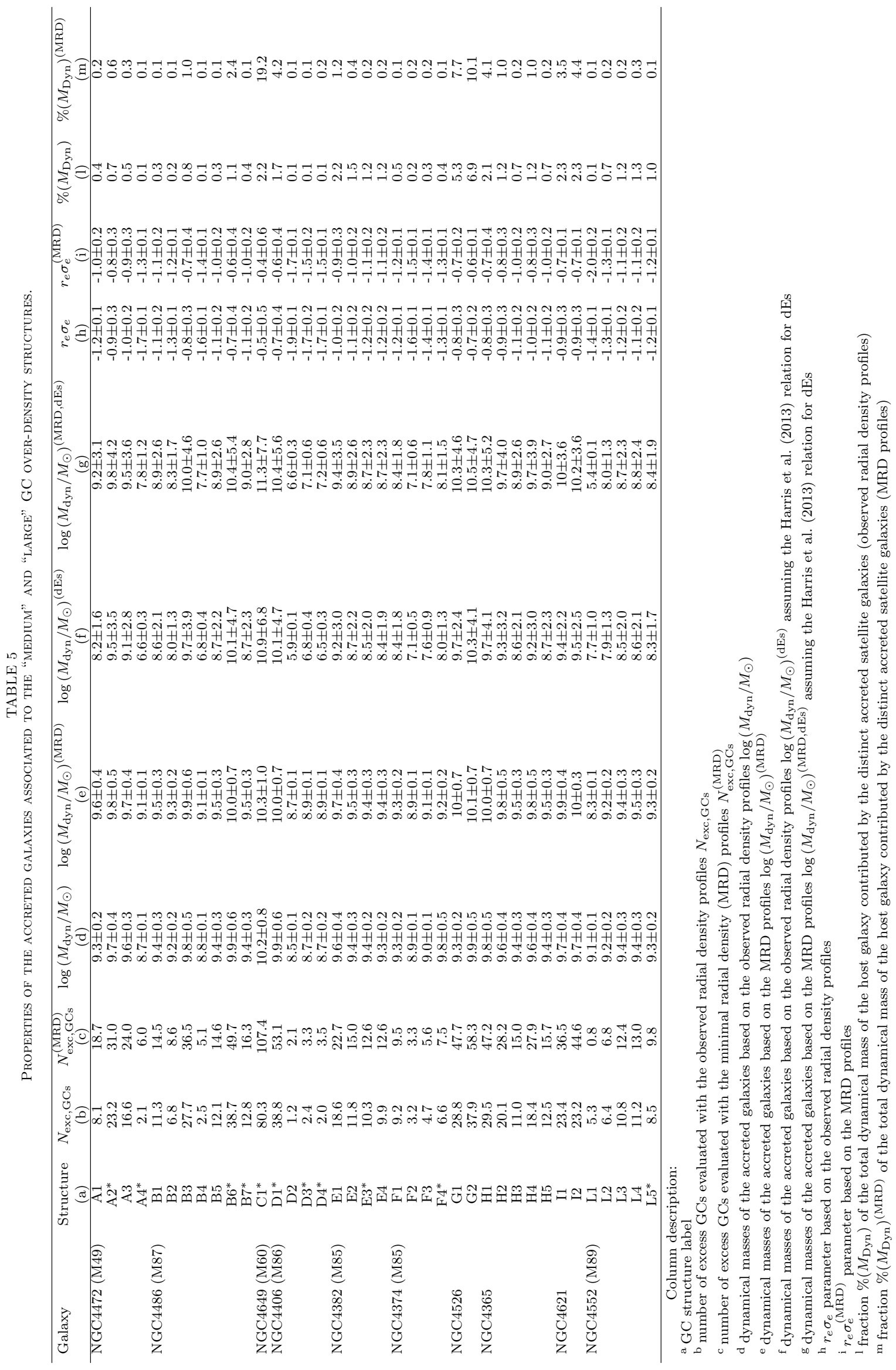




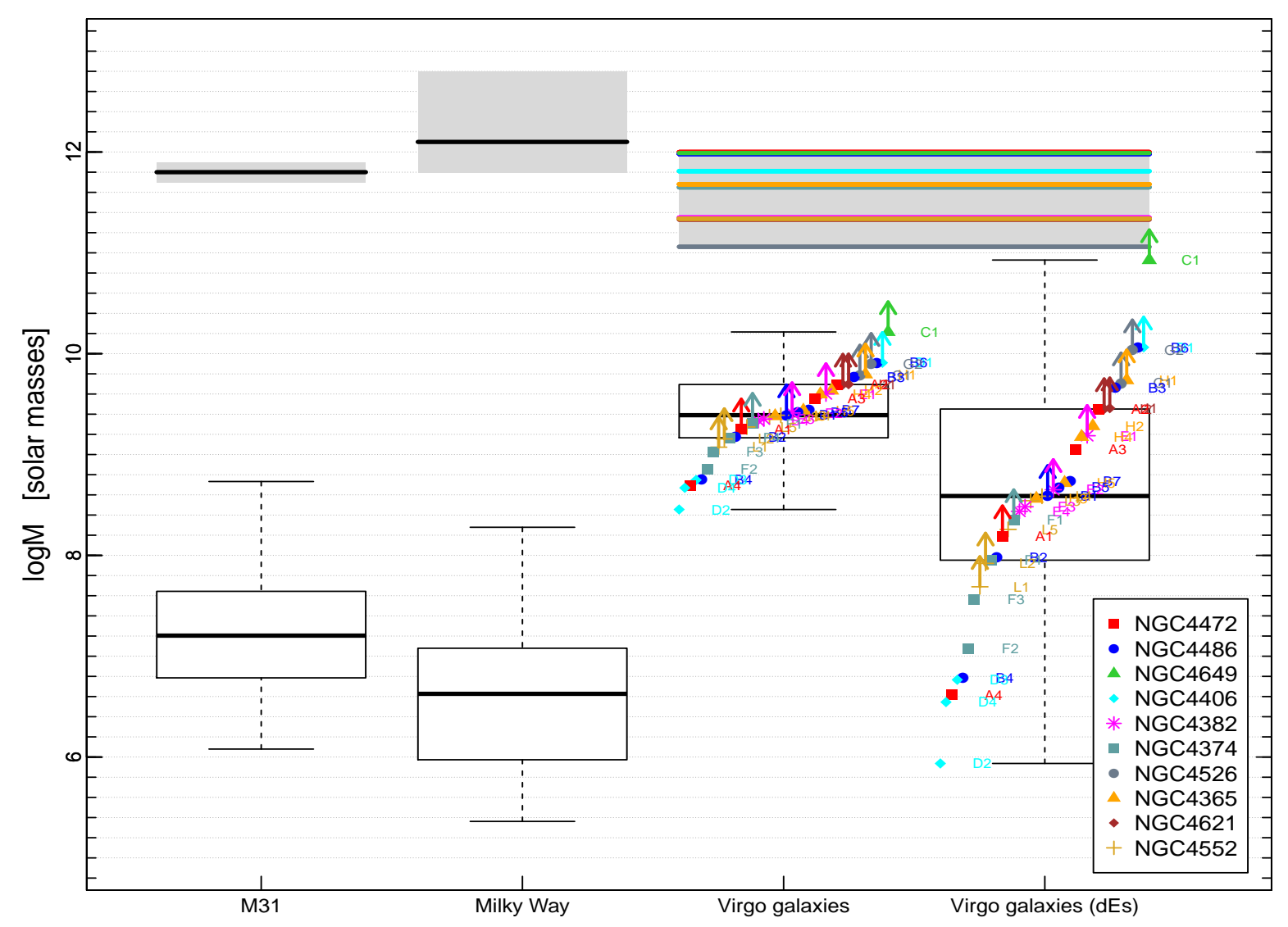

FIG. 15. - Comparison of the distribution of dynamical masses of the satellites of the Virgo galaxies estimated with the method discussed in this paper, with the distributions of masses of the satellite galaxies of M31 and the Milky Way collected by McConnachie (2012). The two boxplots on the right represent the distribution of inferred masses of the Virgo satellite galaxies assuming that the GC over-density structures are due to accretion events. These are determined with the two relations $N_{\mathrm{GCs}}=M_{\mathrm{dyn}}^{1.04 \pm 0.03}$ and $N_{\mathrm{GCs}}=M_{\mathrm{dyn}}^{0.37 \pm 0.09}$ determined by Harris et al. (2013) using all elliptical galaxies of the sample and only dEs, respectively. The grav areas above the boxplots represent the range of mass values for the host galaxies (the masses estimates for M31 are from Evans \& Wilkinson 2000 the interval of masses for the Milky Way is from McMillan (2011), and the single Virgo cluster galaxies are dynamical masses trom Harris et al. 2013). The estimated masses of the individual satellites tor the Virgo cluster galaxies are represented as points with the same symbols and color-coding used in the other plots of the paper. Arrows are used for GC over-density structures that are likely to have been produced by major mergers (as discussed in Section [5.1), and represent lower limits of the masses of the galaxies involved. The horizontal displacement of the points is only shown for the sake of clarity. 
of satellite galaxies or major mergers. While this may be is an oversimplification of the multiple, diverse mechanisms of GC formation at work in massive early-type galaxies (Brodie \& Strader 2006), it may apply at least for some of the GC structures we have detected (see Section 5.2. Below we discuss the possible issues that may affect our conclusions.

\subsubsection{Projection effects}

A concern is the possibility that the observed GC structures are not physical structures, but may results from superposition of GCs, due to projection effects. Such bias cannot be excluded a priori without knowledge of the real three dimensional distribution of GCs around the host galaxy. It is nonetheless reasonable to assume that the importance of projection effects increases towards the center of the galaxy. For instance, Woodley \& Harris (2011) investigated the presence of physical groups of GCs in NGC5128, a giant local elliptical galaxy, and argued that the chance of projection yielding chance two-dimensional groupings of all GCs is high within $10^{\prime}\left(\sim 2 r_{e}\right)$. In our sample, because of the limited ACSVCS spatial coverage (see Figure 12), we cannot exclude that some of the GC structures may be caused or enhanced by projection effects. For instance, streamerlike structure can be generated by three-dimensional GC spatial distributions following the triaxial stellar morphology shown by some elliptical galaxies. However, the risk of detecting unphysical GC structures is mitigated by the fact that most of the large and medium GC structures at distance $d \leq 2 r_{e}$ have complex morphologies that cannot be explained with simple projection effects, assuming a smooth three-dimensional spatial distribution of GCs. Moreover, pure projection effects cannot explain the striking differences observed in the properties of the same GC structures detected in distinct color and luminosity classes (see Section 4), even when we take into account the differences in the radial profile of red and blue GCs.

\subsubsection{Dynamical times}

Another possible issue with our interpretation of the origin of GC structures observed is related to their dynamical evolution. The GC structures located in the outer regions of their host galaxies (A1 and $\mathrm{A} 4$ in NGC4472, C1 in NGC4649 and H1 in NGC4365) might be relatively long-lived, as relaxation times are longer at large galactocentric radii. However, several observations have uncovered evidence of currently ongoing or recent accretion of satellites in the halos of these galaxies (see Section 5.2). The GC structures located closer to the center of the host galaxies, assuming that they are real physical over-densities, ought to be the results of recent events in the assembly history of the host galaxy in order to be still visible today, given that relaxation times are much shorter at small galactocentric radii. This simple reasoning rules out the possibility that these structures have formed in the outskirts of the galaxies and drifted towards their observed positions under the sole effect of the dynamical friction of the host galaxy stellar field.

We can estimate the time required for a single GC orbiting the host galaxy to reach the observed (projected) galactocentric distance from an arbitrary distance under the only effect of dynamical friction. We assume a standard mass for a single GC $M_{G C}=10^{7} M_{\odot}$, consistent with the observed average absolute magnitudes of GCs (Strader et al. 2012), relative stellar circular velocity $v_{\text {circ }} \sim 100 \mathrm{kms}^{-1}$, which is the typical measured values for Virgo cluster galaxies at the largest radius where reliable estimates are available (Cortés et al. 2015) and, as initial distance $r_{i}=1 r_{e}$. The angular distances of the closest dwarfs were converted to physical distances using the galaxy distances by Blakeslee et al. (2009). The dynamical friction time $\Delta t_{\text {fric }}$ of the GC infall has been calculated using equation (7-26) of Binney \& Tremaine (2008), which express the time required for a GC to reach the center of the host galaxy as:

$$
t_{\text {fric }}=\frac{1.17}{\ln \Lambda} \frac{r_{i}^{2} v_{\text {circ }}}{G M}
$$

The final distance $r_{f}$ of the $\mathrm{GC}$ is set to the observed galactocentric distance of the GC over-density, which is a lower limit of the real three-dimensional distance from the center of the galaxy. The $\Delta t_{\text {fric }}$ times range in the 630 Gyr interval for all GC structures in our sample (the structures A1 and A4 in NGC4472, C1 in NGC44649, $\mathrm{H} 1, \mathrm{H} 2, \mathrm{H} 3, \mathrm{H} 4$ and $\mathrm{H} 5$ in NGC4365 and L1 and L2 in NGC4552 were excluded because located at galactocentric distances larger than $1 r_{e}$ ). In the case of spatially extended structures, the final position has been chosen to coincide with the center of mass of the structures. The uncertainties $\sigma\left(\Delta t_{\text {fric }}\right)$ can be as large as $\sim 50 \%$ of the estimated values, with average error $\bar{\sigma}\left(\Delta t_{\text {fric }}\right) \sim 22 \%$. The large times required for these structures to have reached the observed positions under the effect of dynamical friction alone are incompatible with the typically short relaxation times in the center of the galaxy that would have disrupted the GC structures and prevented the observation of the GC overdensity structures. Assuming a fixed deprojected galactocentric distance $r=0.8 r_{e}$, the dynamical friction times are, on average, $\sim 13 \%$ shorter (up to $\sim 40 \%$ in one case), making at least some of the observed GC structures compatible with the effect of internal dynamical evolution only. However, while drifting towards the center of the galaxy under the effect of dynamical friction, the orbits of the GC belonging to a structure would lose coherence due to the differential effect of tidal forces. For instance, Prieto \& Gnedin (2008) found that GCs formed in a satellite halo moving on an initial circular orbit within the satellite halo, deviate significantly from the original orbit in a $\sim 10$ Gyr timeframe (shorter decoherence times are expected if the satellite galaxy has been tidally disrupted before 5 Gyr). GCs formed in the main halo and scattered by accreted satellite halos would deviate significantly from their original circular orbits within $\sim 6$ Gyr (Prieto \& Gnedin 2008) from their formation. For these reasons, internal dynamical evolution alone can be mostly ruled out as explanation of the presence of the GC structures near the center of the galaxies.

A possible formation mechanism for these GC structures is that they are the coherent remains of the GC systems of satellite galaxies falling towards the center of the main galaxy at a large relative velocity. Another possibility is that recent, local formation of new GCs has occurred as a result of a merger. While Côté et al. (1998) and Pipino et al. (2007) found that the radial dis- 
tribution, specific frequency and metallicity of the GC systems of massive elliptical galaxies can be understood by requiring a large fraction of GCs to have formed in satellite galaxies and stripped by the accreting galaxy, the formation of young, metal-rich GCs during mergers is believed to be another significant factor shaping of the observed GC systems of elliptical galaxies (Bekki et al. 2003; Brodie \& Strader 2006, Wang et al. 2013). A detailed modeling of the dynamics of the GC structures progenitors, which is out of the scope of this paper, should also take into account the physical mechanisms acting on the GCs during their crossing of the accreting galaxy potential. These mechanisms would introduce differential effects in the spatial distribution of the observed GCs depending on the relative geometry of the orbit followed by the GC system and the potential of the host galaxy.

\subsubsection{Phase-space counterparts of spatial GC structures}

The physical existence of over-density structures in the projected spatial distribution of GCs can be confirmed if they are also characterized by a specific motion, inherited by the accreted host galaxy or the merger progenitors, which is distinct from the systemic bulk motion of the main host galaxy (e.g. Romanowsky et al. 2012; Coccato et al. 2013; Blom et al. 2012a). The lack of spectroscopic data and kinematic measurements that would confirm the nature of all the GC structures in our sample will only be overcome as new observations become available. However, we stress that the kinematic identification of GC structures is a sufficient but not necessary condition to ascertain the existence of physical groups of GCs originally associated to an accreted satellite galaxy. The kinematic method can only select GC structures with motions significantly different from the systemic velocity of the host galaxy along the line-of-sight direction. GC structures mostly moving transversally to the line-ofsight are missed. Our approach could detect structures no matter what the direction of motion.

\section{SUMMARY AND CONCLUSIONS}

We have studied the 2D distributions of the GC systems of the ten most massive Virgo galaxies, using the GC catalogs extracted from the ACSVCS data (Jordán et al. 2009), and GC catalogs extracted from analogous ACS data by (Strader et al. 2012) and (Blom et al. 2012b). We have estimated the statistical significance of localized spatial structures in these GC 2D distributions, using the KNN method augmented by MC simulations (D'Abrusco et al. 2013). We have applied this method to the $2 \mathrm{D}$ distribution all the GCs in each galaxy and also to the classes of red, blue, low-L and high-L GCs (Section 4). Our results can be summarized as follows:

- GCs over-density structures are common in the sample of galaxies studied. We observed a total of $229 \mathrm{GC}$ structures in the residual maps of the spatial distribution of all GCs in the ten galaxies, and 42 significant, spatially extended GC structures classified as "medium" or "large" structures, i.e. structures formed by groups of 5 to 19 adjacent pixels or formed by 20 or more pixels with total significance larger than $4 \sigma$, respectively (see Section 4). Most of these GC structures are also visible in the residual maps for red/blue and/or high-L/low-L GC subsamples. The physical sizes of the large and intermediate GC structures range between $\sim 1$ to $\sim 25 \mathrm{kpc}$.

- The spatial distribution of the small over-density structures is radially and azimuthally homogeneous (between $1^{\prime}$ and $2^{\prime}$ ). However, with the present data we cannot exclude that some of these small structures may be due to statistical noise.

- The medium and large structures may be related to the accretion of smaller satellite galaxies. This effect is stronger when the area of the GC structures is considered. However, the spatial coverage of our sample is far from complete at larger radii.

- The large, coherent spatial structures, which tend to be aligned along the major axis of the host galaxies and are clearly visible in the spatial distribution of red GCs, could be the result of major mergers, as predicted by simulations (see Bekki et al. 2002). The medium-size structures may be related to the accretion of smaller satellite galaxies.

- The smaller spatial structures (accounting for only $\sim 14 \%$ of the total area of the GC over-density structures observed) are observed to be more likely to be located along the direction of the minor axis of the host galaxy than the large structures than on the major axis. No significant differences are observed in their color and luminosity distributions.

- Large, spatially complex GC structures, mostly aligned along the major axis of the host galaxies, are more likely to be found in low-density regions within the Virgo cluster than in the high-density areas. While the red large GCs structures are, on average, more significant than structures in the spatial distribution of all GCs (Section 5.1), this effect is stronger in the lower-density environment, suggesting the relatively recent mergers have determined the GC spatial distribution in these galaxies. Additional observations will be necessary to rule out observational biases.

- Using the total number of excess GCs associated to the large and medium GCs structures as a proxy of the size of the whole GC system, we have calculated the range of dynamical masses of the satellites using the relations from Harris et al. (2013). We found that masses of the satellites estimated with our method are significantly larger than the observed masses of the satellites in the Milky Way and M31, suggesting that the environment plays an important role in the assembly of galaxies by accretion of satellites. The estimated masses of the progenitors are too small to be compatible with assembly histories involving recent major mergers, especially in the case of the host galaxies discussed in Section 5.4. We argue that, in case of major mergers, these disagreements could be reconciled with a more detailed description of the evolution of GC systems and the relation between size of the GC system and the properties of the host galaxy. 
We also note that non-equal mass wet mergers with larger mass ratios could also be responsible for significant GC formation.

We have proposed a simple mechanism responsible for the formation of these GC structures that invokes either small accretion of satellite galaxies or, in the case of the largest, spatially extended structures, major mergers, and used it to derive fundamental properties of the potential progenitor galaxies (Section 5.5).

Our results show that 2D over-density structures in the spatial distribution of GCs in massive early-type galaxies are a common phenomenon, but we are currently limited by the lack of deep, high spatial resolution data reaching the outskirts of the host galaxies. New HST observations complementing the ACSVCS data in the outer regions of the Virgo galaxies are necessary to fully determine the characteristics of the $2 \mathrm{D}$ spatial distribution of GCs. Moreover, GCs catalogs covering larger galactocentric baseline would be useful to compare our results with data from other tracers of the dynamics and evolution of the host galaxy, like GCs and Planetary Nebulae kinematics. The events in the assembly history of the host galaxy that, according to our model, may have generated the observed GC structures, should also leave evident signatures in the dynamics of these systems. Unfortunately, kinematic data for homogeneous, large samples of GCs located within the inner region of the host galaxies that could validate our assumptions are difficult to obtain.

Constraining the evolution of massive galaxies also requires synergy between observations and simulations.
Fully cosmological simulations of galaxy mergers are now being extended to resolve GC-sized objects (Mayer 2014, private communication) and to determine the effect of hierarchical evolution on the spatial distribution of the GC systems. This new generation of simulations will provide a unique testbed for the formation and evolution of GCs with unprecedented resolution and physical detail, producing GC parameters directly comparable with the observations. The comparison of the features of the observed 2D GC structures with the simulated data will assess how the observed 2D GC structures can be used to infer past merging/accretion history of the host galaxies. The study of the GC over-density structures and its comparison with cosmological simulations that include GC formation will represent a powerful, game-changing combination of tools to advance the understanding of how galaxies grow and evolve.

The authors thank the anonymous referee for insightful comments that have helped to improve the manuscript. The authors also thank Marta Volonteri for useful comments to the manuscript. R. D'A. acknowledges the SI competitive research grant "A New Astro-Archeology Probe of the Merging Evolution of Galaxies" for support. R. D'A.'s work was also partially supported by the Chandra X-ray Center (CXC), which is operated by the Smithsonian Astrophysical Observatory (SAO) under NASA contract NAS8-03060. AZ acknowledges funding from the European Research Council under the European Union's Seventh Framework Programme (FP/20072013)/ERC Grant Agreement n. 617001.

\section{APPENDIX}

\section{COMPARISON WITH OTHER DENSITY ESTIMATION METHODS}

In order to confirm the effectiveness of our method and its ability to detect over-density structures in the projected spatial distribution of GCs, we have compared the residual maps obtained with our KNN-based technique with residual maps derived with a multidimensional Gaussian Mixture Model (GMM) technique (Dempster et al. 1977) and the Kernel Density Estimation (KDE) method (Rosenblatt 1956, Parzen 1962).

The GMM method approximates the density function of the observed points with a combination of multiple, independent, multi-dimensional Gaussian distributions, where each component represents a different group or subpopulation. In this paper, we have used the Mclust implementation (Fraley et al. 2012) of the GMM technique, written in $R$ (R Core team 2014). In this implementation, the number of mixture model Gaussian components can be either specified by the user or determined automatically by the algorithm with an EM approach. $R$ is an open-source free statistical environment developed under the GNU GPL (http://www.r-project.org). An application of the Mclust algorithm to the problem of the detection of substructures in the velocity distribution of a sample of galaxies is discussed by Einasto et al. (2012).

The KDE method is a non-parametric density estimation technique which reconstructs the unknown density function of a random variable using a kernel with a typical smoothing parameter. This parameter, the bandwidth, is estimated locally and depends on the local topology of the distribution of points whose density function is reconstructed.

We have applied the KNN, GMM and KDE methods for the determination of the density maps to the observed distribution of ACSVCS GCs in NGC4472 (see Section 4.1), and to a simulated distribution of GCs where distinct components are visible. Density maps of the two GCs distributions have been determined in both cases. The other steps for the calculation of the residual maps from the density of the observed GCs and the model simulated GC distribution were unchanged.

\section{Spatial distribution of GCs in NGC4472}

The results of the application of the three density estimation techniques to the spatial distribution of the observed GCs distribution in NGC4472 are shown in Figure 16. The upper left plot shows the residual maps obtained with the KNN method $(K=9)$ described in Section 4.1, the upper right plot is the residual map obtained using the KDE, while the lower panels show the residual maps obtained using the Mclust method where the number of components of the mixture model is determined automatically by the algorithm (left), or fixed to the optimal value 5 (right). The

\footnotetext{
5 We have derived the residual maps for number of components
} varying between 3 and 30 , and selected the map the most closely 

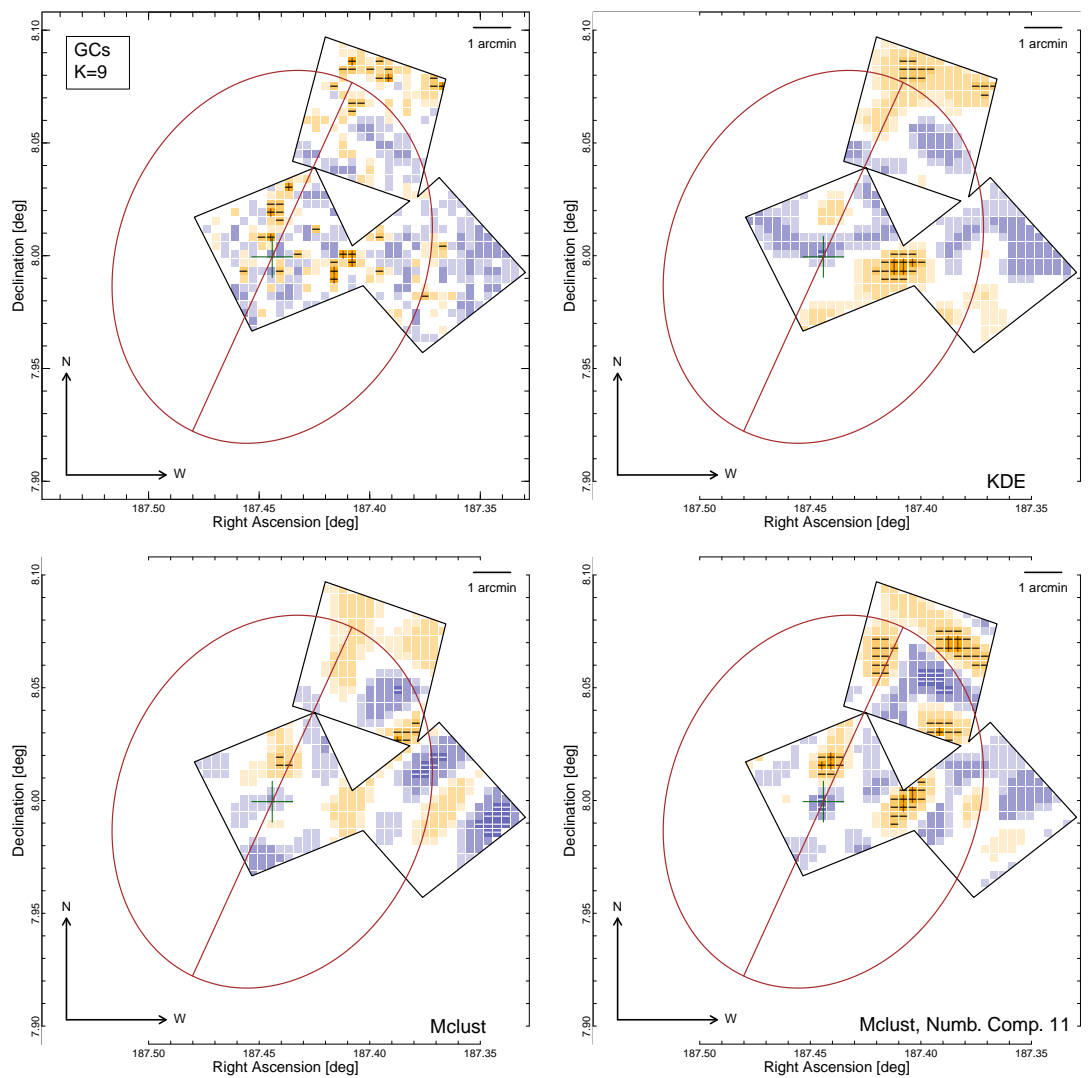

FIG. 16. - Residual maps of the GC distribution in NGC4472 obtained with different density estimation methods: upper left) KNN with $K=9$; upper right) $\mathrm{KDE}$; lower left) Mclust with number of mixture model components automatically determined; lower right) Mclust with optimal number of components fixed to 11.

four residual maps are in substantial agreement on the overall number and positions of the GC structures detected by the KNN method, while the spatial extension varies significantly. The KDE and Mclust methods produce "smoother" and more spatially extended structures than the KNN method.

\section{Simulated spatial distribution of GCs}

We have also applied the KNN, KDE and Mclust methods to a simulated distribution of GCs (Figure 17) consisting in three different components: an azimuthally smooth distribution of GCs with radial positions randomly drawn from a power-law peaking in the center of the galaxy; three clusters, modeled as two-dimensional Gaussians with different variances along the R.A. and Dec. axes, radial and azimuthal positions relative to the center of the galaxy, and one "streamer", modeled as a uniform azimuthal and radial distribution of GCs within a circular sector with 40deg aperture. The total number of simulated GCs is 1750, split in 1500 GCs belonging to the smooth distribution, 100 GCs in the "streamer" and 50 GCs for each cluster. The total number of GCs and the number of GCs in each spatial component have been set to yield average GC densities and GC density contrasts in the over-density structures comparable to the observed values in the Virgo galaxies investigated in this paper (see Table 2 for comparison). As a result, the number of excess GCs per pixels in each spatial structure of the simulated distribution is similar to the average number of excess GCs in the structures observed in the galaxies investigated. The spatial distribution of the simulated components are color-coded in the upper left panel in Figure 17. As in the case of the NGC4472 GC system, the residuals maps produced with the three methods are similar: the two clusters $\mathrm{E}$ and $\mathrm{N}-\mathrm{W}$ of the center of the galaxy are recovered in each map as well as the streamer (which, in the case of the KDE, has a rounded shape reflecting the use of a Gaussian as kernel function). The third cluster located just $\mathrm{S}$ of the center of the galaxy is associated to lesser over-density structures in the KNN and Mclust residual maps but it is missed by the KDE. Both the KDE and Mclust maps show artifacts structures located in the corners of the field and due to border effects, which are missing in the map produced by the KNN.

\section{Conclusions}

The three methods recover the same GC structures in both examples, with similar spatial and statistical properties. The shapes of the over-densities reconstructed with the GMM and KDE techniques, in general, have smoother boundaries than those of the KNN-determined structures because the two former methods tend to spread the small-scale over-densities (often driven by GC excesses in one or few pixels) over larger areas. Moreover, GMM and KNN "bridge 

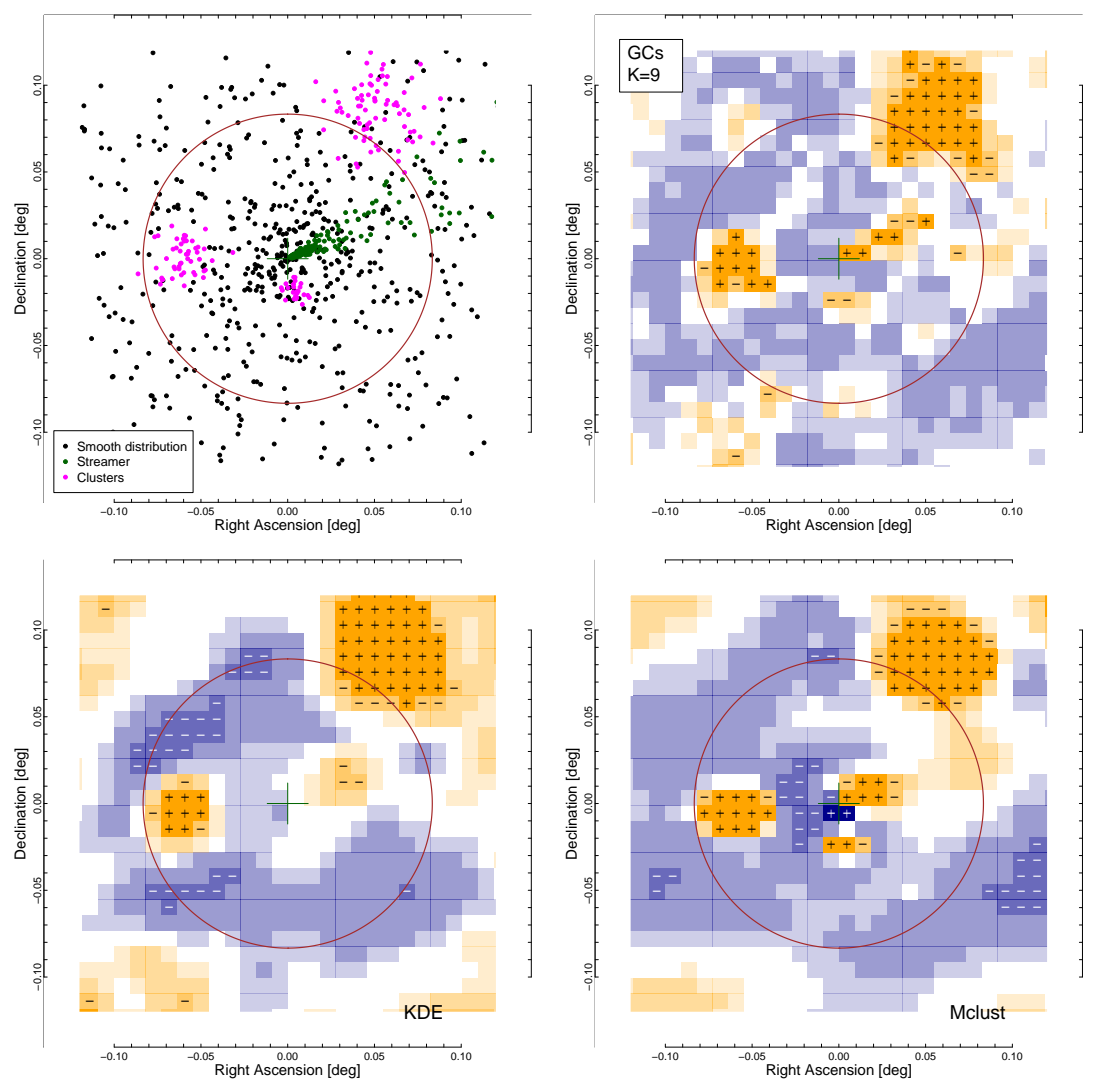

FIG. 17. - Scatterplot of a simulated distribution of GCs with three distinct components (upper left) and residual maps obtained with the $K=9 \mathrm{KNN}$ method (upper right), the KDE (lower left) and the Mclust method with number of mixture model components automatically determined (lower right).

over" sharp pixel-by-pixel changes in the GC density, and the reconstructed final shapes are not representative, on spatial scales of few pixels, of the real geometry of the structures. The significances of the structures have been measured independently in the residual maps shown in Figures 16 and 17 as described in Section 3 . Overall, the significances of all GC structures in both GC distributions differ by less than $1.8 \sigma$ and $0.8 \sigma$ respectively, with negligible overall bias in the their distribution. The overall similarity of the significances indicates that the KNN method provides robust estimates of the real statistical significances of the GC structures. The small fluctuations measured are likely due to the different spatial extensions of the GC structures detected in the different residual maps. For instance, the lack of $\geq 3 \sigma$ pixels in the map of the NGC 4472 GCs spatial distribution in Figure 16 produced with the unconstrained Mclust algorithm is balanced by the larger areas occupied by the structures. The combined effect of singe pixels significance and total extension and shape of the structures produced similar statistical significances for all methods tested.

Our conclusions is that, overall, the properties (location, significance and shape) of the GC residual structures obtained with the KNN method, are robust and in substantial agreement with the features of the GC structures detected using different density estimation methods.

\section{REFERENCES}

Arnold, J. A., Romanowsky, A. J., Brodie, J. P., et al. 2014, ApJ, 791,80

Ashman, K. M., \& Zepf, S. E. 1992, ApJ, 384, 50

Ashman, K. M., \& Zepf, S. E. 1998, Globular cluster systems / Keith M. Ashman, Stephen E. Zepf. Cambridge, U. K. ; New York : Cambridge University Press, 1998. (Cambridge astrophysics series ; 30) QB853.5 .A84 1998 (\$69.95)

Bassino, L. P., Faifer, F. R., Forte, J. C., et al. 2006, A\&A, 451, 789

Bekki, K., \& Shioya, Y. 1997, ApJ, 478, L17

Bekki, K., \& Shioya, Y. 1998, ApJ, 497, 108

Bekki, K., Forbes, D. A., Beasley, M. A., \& Couch, W. J. 2002, MNRAS, 335, 1176

Bekki, K., Forbes, D. A., Beasley, M. A., \& Couch, W. J. 2003, MNRAS, 344, 1334

Bekki, K., Beasley, M. A., Brodie, J. P., \& Forbes, D. A. 2005, MNRAS, 363, 1211
Belokurov, V., Zucker, D. B., Evans, N. W., et al. 2006, ApJ, 647, L111

Binggeli, B., Sandage, A., \& Tammann, G. A. 1985, AJ, 90, 1681

Binney, J., \& Tremaine, S. 2008, Galactic Dynamics: Second Edition, by James Binney and Scott Tremaine. ISBN 978-0-691-13026-2 (HB). Published by Princeton University Press, Princeton, NJ USA, 2008

Blakeslee, J. P., Jordán, A., Mei, S., et al. 2009, ApJ, 694, 556

Blom, C., Forbes, D. A., Brodie, J. P., et al. 2012, MNRAS, 426, 1959

Blom, C., Spitler, L. R., \& Forbes, D. A. 2012, MNRAS, 420, 37

Blom, C., Forbes, D. A., Foster, C., Romanowsky, A. J., \& Brodie, J. P. 2014, MNRAS, 439, 2420

Bogdán, Á., Forman, W. R., Kraft, R. P., et al. 2012, ApJ, 755, 25 Bonfini, P., Zezas, A., Birkinshaw, M., et al. 2012, MNRAS, 421, 2872

Bower, G. A., Green, R. F., Danks, A., et al. 1998, ApJ, 492, L111 Brodie, J. P., \& Strader, J. 2006, ARA\&A, 44, 193 
Coccato, L., Arnaboldi, M., \& Gerhard, O. 2013, MNRAS, 436, 1322

Cohen, J. G., Blakeslee, J. P., \& Ryzhov, A. 1998, ApJ, 496, 808

Cohen, J. G., Blakeslee, J. P., \& Côté, P. 2003, ApJ, 592, 866

Côté, P., Marzke, R. O., \& West, M. J. 1998, ApJ, 501, 554

Côté, P., Blakeslee, J. P., Ferrarese, L., et al. 2004, ApJS, 153, 223

Cortés, J. R., Kenney, J. D. P., \& Hardy, E. 2015, ApJS, 216, 9

D'Abrusco, R., Fabbiano, G., \& Brassington, N. J. 2014, ApJ, 783,19

D'Abrusco, R., Fabbiano, G., Mineo, S., et al. 2014, ApJ, 783, 18

D'Abrusco, R., Fabbiano, G., Strader, J., et al. 2013, ApJ, 773, 87

Das, P., Gerhard, O., Mendez, R. H., Teodorescu, A. M., \& de Lorenzi, F. 2011, MNRAS, 415, 1244

Davies, R. L., Kuntschner, H., Emsellem, E., et al. 2001, ApJ, 548, L33

Dempster, A., Laird, N., Rubin, D.: Maximum likelihood from incomplete data via the EM algorithm, J. Royal Stat. Soc. 39(1), 138 (1977)

de Vaucouleurs, G., de Vaucouleurs, A., Corwin, H. G., Jr., et al. 1991, S\&T, 82, 621

Di Matteo, T., Springel, V., \& Hernquist, L. 2005, Nature, 433, 604

Dirsch, B., Richtler, T., Geisler, D., et al. 2003, AJ, 125, 1908

Dirsch, B., Schuberth, Y., \& Richtler, T. 2005, A\&A, 433, 43

di Serego Alighieri, S., Bianchi, S., Pappalardo, C., et al. 2013, A\&A, 552, AA8

Dressler, A. 1980, ApJ, 236, 351

Einasto, M., Vennik, J., Nurmi, P., et al. 2012, A\&A, 540, AA123

Evans, N. W., \& Wilkinson, M. I. 2000, MNRAS, 316, 929

Fall, S. M., \& Zhang, Q. 2001, ApJ, 561, 751

Ferrarese, L., Côté, P., Jordán, A., et al. 2006, ApJS, 164, 334

Forbes, D. A., Brodie, J. P., \& Grillmair, C. J. 1997, AJ, 113, 1652

Fraley, C., Raftery, A. E., Murphy, T. B., and Scrucca, L. 2012, Mclust Version 4 for R: Normal Mixture Modeling for Model-Based Clustering, Classification, and Density Estimation, Technical Report No. 597, Department of Statistics, University of Washington.

Georgiev, I. Y., Puzia, T. H., Goudfrooij, P., \& Hilker, M. 2010 MNRAS, 406, 1967

Gomez, H. L., Baes, M., Cortese, L., et al. 2010, A\&A, 518, LL45

Graves, G. J., \& Faber, S. M. 2010, ApJ, 717, 803

Hargis, J. R., \& Rhode, K. L. 2012, AJ, 144, 164

Harris, W. E., \& van den Bergh, S. 1981, AJ, 86, 1627

Harris, W. E. 1986, AJ, 91, 822

Harris, W. E., Harris, G. L. H., \& Alessi, M. 2013, ApJ, 772, 82

Ibata, R. A. Gilmore, G., \& Irwin, M. J. 1994, Nature, 370, 194

Janowiecki, S., Mihos, J. C., Harding, P., et al. 2010, ApJ, 715, 972

Mateo, M., Olszewski, E. W., Pryor, C., Welch, D. L., \& Fischer, P. 1993, AJ, 105, 510

McConnachie, A. W. 2012, AJ, 144, 4

Mineo, S., Fabbiano, G., D'Abrusco, R., et al. 2014, ApJ, 780, 132
Jordán, A., Blakeslee, J. P., Peng, E. W., et al. 2004, ApJS, 154, 509

Jordán, A., McLaughlin, D. E., Côté, P., et al. 2007, ApJS, 171, 101

Jordán, A., Peng, E. W., Blakeslee, J. P., et al. 2009, ApJS, 180, 54

Lane, R. R., Salinas, R., \& Richtler, T. 2013, A\&A, 549, A148

Lee, M. G., Hwang, H. S., Park, H. S., et al. 2008, ApJ, 674, 857

McConnachie, A. W., Irwin, M. J., Ibata, R. A., et al. 2009, Nature, 461,66

McMillan P. J., 2011, MNRAS, 414, 2446

McNamara, B. R., Sancisi, R., Henning, P. A., \& Junor, W. 1994, AJ, 108, 844

Parzen, E., 1962, On Estimation of a Probability Density Function and Mode, The Annals of Mathematical Statistics 33 (3): 1065

Peñarrubia, J., Walker, M. G., \& Gilmore, G. 2009, MNRAS, 399, 1275

Peng, E. W., Jordán, A., Côté, P., et al. 2008, ApJ, 681, 197

Perrett, K. M., Stiff, D. A., Hanes, D. A., \& Bridges, T. J. 2003 ApJ, 589, 790

Pierce, M., Bridges, T., Forbes, D. A., et al. 2006, MNRAS, 368, 325

Pipino, A., Puzia, T. H., \& Matteucci, F. 2007, ApJ, 665, 29

Pota, V., Forbes, D. A., Romanowsky, A. J., et al. 2013, MNRAS, 428 ,

Prieto, J. L., \& Gnedin, O. Y. 2008, ApJ, 689, 919

$\mathrm{R}$ Core Team, 2014, R: A Language and Environment for Statistical Computing, Vienna, Austria, http://www.R-project.org/ \& Larsen, S. S. 2007, MNRAS, 377, 1772

Rhode, K. L., \& Zepf, S. E. 2001, AJ, 121, 210

Rhode, K. L., \& Zepf, S. E. 2004, AJ, 127, 302

Romanowsky, A. J., Strader, J., Brodie, J. P., et al. 2012, ApJ, 748,29

Rosenblatt, M., 1956, Remarks on Some Nonparametric Estimates of a Density Function, The Annals of Mathematical Statistics 27 (3): 832

Schweizer, F., Seitzer, P., Faber, S. M., et al. 1990, ApJ, 364, L33

Searle, L., \& Zinn, R. 1978, ApJ, 225, 357

Smith, R. J., Lucey, J. R., Price, J., Hudson, M. J., \& Phillipps, S. 2012, MNRAS, 419, 3167

Springel, V., White, S. D. M., Jenkins, A., et al. 2005, Nature, 435,629

Strader, J., Romanowsky, A. J., Brodie, J. P., et al. 2011, ApJS, 197,33

Strader, J., Fabbiano, G., Luo, B., et al. 2012, ApJ, 760, 87

Teodorescu, A. M., Méndez, R. H., Bernardi, F., et al. 2011, ApJ, 736,65

Wang, Q., Peng, E. W., Blakeslee, J. P., et al. 2013, ApJ, 769, 145

White, S. D. M., \& Rees, M. J. 1978, MNRAS, 183, 341

Wolf, J., Martinez, G. D., Bullock, J. S., et al. 2010, MNRAS, 406,1220

Woodley, K. A., \& Harris, W. E. 2011, AJ, 141, 27 